\title{
CHARACTERIZING ARTIN STACKS
}

\author{
SHARON HOLLANDER
}

\begin{abstract}
We study properties of morphisms of stacks in the context of the homotopy theory of presheaves of groupoids on a small site $\mathcal{C}$. There is a natural method for extending a property $P$ of morphisms of sheaves on $\mathcal{C}$ to a property $\mathcal{P}$ of morphisms of presheaves of groupoids. We prove that the property $\mathcal{P}$ is homotopy invariant in the local model structure on $P(\mathcal{C}, \mathcal{G r p d})_{L}$ when $P$ is stable under pullback and local on the target. Using the homotopy invariance of the properties of being a representable morphism, representable in algebraic spaces, and of being a cover, we obtain homotopy theoretic characterizations of algebraic and Artin stacks as those which are equivalent to simplicial objects in $\mathcal{C}$ satisfying certain analogues of the Kan conditions.

The definition of Artin stack can naturally be placed within a hierarchy which roughly measures how far a stack is from being representable. We call the higher analogues of Artin stacks $n$-algebraic stacks, and provide a characterization of these in terms of simplicial objects. A consequence of this characterization is that, for presheaves of groupoids, $n$-algebraic is the same as 3-algebraic for all $n \geq 3$.

As an application of these results we show that a stack is $n$-algebraic if and only if the homotopy orbits of a group action on it is.
\end{abstract}

\section{INTRODUCTION}

In the ground breaking work of Deligne and Mumford [DM], stacks were invented in order to prove the irreducibility of the moduli of curves. One of the key innovations enabling them to prove this was the ability to extend standard definitions of properties of morphisms between schemes, such as étale, smooth, etc., to well defined properties of morphisms between stacks.

From our point of view, stacks are best understood as fibrant objects in the local model structure on the category of presheaves of groupoids on a small site $\mathcal{C}$, which is denoted $P(\mathcal{C}, \mathcal{G r p d})_{L}$. Stacks are the presheaves which satisfy the analogue of the sheaf condition when the homotopy theory of groupoids is appropriately taken into account (see [H1]). Unlike sheaves of sets, the subcategory of stacks does not have good properties like existence of limits and colimits, though it does naturally have a notion of equivalence which is modeled nicely by the homotopy category $H o\left(P(\mathcal{C}, \mathcal{G r p d})_{L}\right)$. Studying stacks in this context means working with and in the entire model category $P(\mathcal{C}, \mathcal{G r p d})_{L}$.

The aim of this paper is first to define properties of morphisms in the context of the homotopy theory of stacks and then to apply this to give a generalization and homotopy theoretic characterization of Artin stacks and higher order analogues.

We now give a more detailed description of the contents of the paper.

For $P$ a property of morphisms between schemes, Deligne and Mumford defined a morphism between stacks $\mathcal{M}^{\prime} \stackrel{f}{\longrightarrow} \mathcal{M}$ to have property $P$ if for every map of

1991 Mathematics Subject Classification. Primary 14A20 ; Secondary 18G55, 55U10. 
schemes $X \rightarrow \mathcal{M}$ the 2-category pullback $X \times{ }_{\mathcal{M}}^{2} \mathcal{M}^{\prime}$ is a scheme, and the projection map to $X$ has property $P$. One can prove (see [H3, Lemma 2.2]) that in our homotopy theoretic context, the 2-category pullback is a model for the homotopy pullback in the model category $P(\mathcal{C}, \mathcal{G} r p d)_{L}$. It follows that we can define properties of morphisms in $P(\mathcal{C}, \mathcal{G r p d})_{L}$ as in $[\mathrm{DM}]$ only replacing 2-category pullback with homotopy pullback, that is, we require that the projection map $X \times{ }_{\mathcal{M}}^{h} \mathcal{M}^{\prime} \rightarrow X$ be weakly equivalent to a map of representables satisfying property $P$.

In many cases, the property that one would like to extend is not only defined for maps of representables but for more general sheaves. For instance, if $\mathcal{C}$ is the category of affine schemes one would like to consider the collection of morphisms corresponding to open subschemes. This is most naturally defined as a property of morphisms between sheaves on $\mathcal{C}$. Any property of morphisms between sheaves on $\mathcal{C}$ with representable target can naturally be extended to a property of morphisms in $P(\mathcal{C}, \mathcal{G r p d})_{L}$, as follows:

Definition 1.1 (Definition 2.6). Let $P$ be a property of morphisms in $S h(\mathcal{C})$ with representable target. We say that $f: \mathcal{M} \rightarrow \mathcal{N} \in P(\mathcal{C}, \mathcal{G} r p d)_{L}$ satisfies property $\mathcal{P}$ if for all maps $X \rightarrow \mathcal{N}$ with $X \in \mathcal{C}$, the map $X \times{ }_{\mathcal{N}}^{h} \mathcal{M} \rightarrow X$ is weakly equivalent to a map in $\mathrm{Sh}(\mathcal{C})$ which satisfies property $P$.

Both philosophically and practically, this extended property $\mathcal{P}$ is only sensible if it is homotopy invariant in $P(\mathcal{C}, \mathcal{G r p d})_{L}$. We prove that $\mathcal{P}$ is a homotopy invariant when $P$ is stable under pullback and local on the target (see Theorem 2.8). These hypotheses are very natural and hold for many of the usual properties. However it is not always trivial to prove that they hold. An example of this is when $\mathcal{C}=\mathcal{A} f f_{f p q c}$ is the site of affine schemes in the flat topology and the property is being a map between representables. In this case, the fact that this property is local on the target amounts to faithfully flat descent.

When $P$ is the property of being a map between representables, $\mathcal{P}$ is called being a representable morphism. In [H3] we extended Deligne and Mumford's definition of algebraic stack to an arbitrary site in the following way.

Definition 1.2 (Definition 2.11). We say that $\mathcal{M} \in P(\mathcal{C}, \mathcal{G r p d})$ is generalized algebraic if

(i) Its diagonal $\mathcal{M} \rightarrow \mathcal{M} \times \mathcal{M}$ is representable,

(ii) There is a cover $X \rightarrow \mathcal{M}$ with $X \in \mathcal{C}$.

With the above understanding of properties of morphisms we can give a vastly streamlined proof of the characterization of algebraic stacks originally proven in [H3].

Theorem 1.3 (Theorem 2.15). Let $\mathrm{C}$ be a site which is local and satisfies descent for morphisms. Then $\mathcal{M}$ is a generalized algebraic presheaf of groupoids if and only if there is a weak equivalence

$$
\left(X_{o}, X_{m}\right) \stackrel{\sim}{\longrightarrow} \mathcal{M} \in P(\mathcal{C}, \mathcal{G r p d})_{L}
$$

where

- $\left(X_{o}, X_{m}\right)$ is a groupoid object in $\mathcal{C}$,

- the domain map $X_{m} \rightarrow X_{o}$ is a cover.

In section 3 we consider the theory of algebraic spaces in preparation for the following section where Artin stacks are introduced. We recall both Artin and 
Knutson's definitions of algebraic spaces and compare them with another abstract definition: we call a sheaf of sets on $\mathcal{C}$ an algebraic space if it is also an algebraic stack in the sense of Definition 2.11. We then present conditions on the site $\mathcal{C}$ which guarantee that the property of having domain an algebraic space is local on the target. We call this sites stackable (see Definition 3.10). These hold for most familiar topologies on schemes. We also give conditions under which a property $P$ can be extended to a well defined property of morphisms with domain an algebraic space which is stable under pullback and local on the target. We call such properties covering invariant (see Definition 3.13). In this case we can extend this property to a homotopy invariant property of morphisms in $P(\mathcal{C}, \mathcal{G r p d})_{L}$ - the property of being representable in algebraic spaces with property $\mathcal{P}$ (Definition 3.15).

In section 4 we recall Artin's definition of stack and present an abstraction of this definition to an arbitrary site $\mathcal{C}$ and covering invariant property $P$.

Definition 1.4 (Definition 4.2). We say that $\mathcal{M} \in P(\mathcal{C}, \mathcal{G r p d}$ ) is $P$-Artin if

- its diagonal is representable in algebraic spaces, and

- there is a map $X \rightarrow \mathcal{M}$ from a representable $X$ which is locally essentially surjective and has property $\mathcal{P}$ (in the sense of Definition 3.15).

When $\mathcal{C}$ is locally finite type schemes in the étale topology and $P$ is the property of being a smooth morphism we explain how this gives back Artin's original definition. Next we give a characterization of $P$-Artin stacks in terms of simplicial diagrams. Recall that $\pi_{\text {oid }}$ denotes the fundamental groupoid functor $s \delta e t \rightarrow$ Grpd, which can be applied to a presheaf of simplicial sets to obtain a presheaf of groupoids.

Theorem 1.5 (Theorem 4.5). Let $\mathrm{C}$ be a stackable site and $P$ be a covering invariant property. A presheaf of groupoids $\mathcal{M}$ is $P$-Artin if and only if there is a weak equivalence

$$
\pi_{\text {oid }} X \stackrel{\sim}{\longrightarrow} \mathcal{M}
$$

where $X_{\bullet} \in \mathcal{C}_{\bullet}$ is a simplicial object in $\mathcal{C}$ satisfying

(i) $X_{\bullet}$ is a 2-coskeleton.

(ii) $d_{0}, d_{1}: X_{1} \rightarrow X_{0}$ have property $P$,

(iii) The Kan maps $X_{2} \rightarrow X_{\bullet}^{\Lambda^{2}}$ are covers for $i=0,1,2$.

(iv) The Kan maps $X_{k} \rightarrow X_{\bullet}^{\Lambda_{i}^{k}}$ are isomorphisms for $k>2$ and all $i$.

One may consider a presheaf of groupoids $\mathcal{M}$ to be a presheaf of simplicial sets, via the nerve embedding $\mathcal{G r p d} \rightarrow$ sSet. In this context, working in the local model structure on presheaves of simplicial sets $P(\mathcal{C}, s \mathcal{S} e t)_{L}$ (see [DI]), we have the following equivalent statement, which we prove as a Corollary of the Theorem above.

Corollary 1.6. A presheaf of groupoids $\mathcal{M}$ is $P$-Artin if and only if there is a weak equivalence

$$
X_{\bullet} \stackrel{\sim}{\longrightarrow} \mathcal{M} \in P(\mathcal{C}, s \mathcal{S e t})_{L}
$$

where $X_{\bullet} \in \mathcal{C}_{\bullet}$ satisfying the hypothesis of Theorem 4.5.

When the property $P$ is that of being a cover, the definition of $P$-Artin indicates a natural inductive definition of $n$-algebraic stacks: A 0 -algebraic stack is a representable sheaf, a 1-algebraic stack is an algebraic stack as in Definition 2.11, 
a 2 -algebraic stack is a $P$-Artin stack with $P$ the property of being a cover, and, in general,

- we say that $\mathcal{M} \in P(\mathcal{C}, \mathcal{G r p d})$ is $n$-algebraic if there is $X \in \mathcal{C}$ and a cover $X \rightarrow \mathcal{M}$ which is representable in $(n-1)$-algebraic spaces $((n-1)$-algebraic stacks which are weakly equivalent to sheaves of sets).

We prove an analogous characterization of $n$-algebraic stack (Theorem 4.14). A somewhat surprising consequence of this characterization is that the hierarchy we have described for algebraic stacks ends at $n=3$, i.e. if $\mathcal{M}$ is $n$-algebraic with $n>3$ then $\mathcal{M}$ is in fact 3 -algebraic.

Theorem 1.7. (Theorem 4.14). Let $\mathcal{C}$ be a stackable site in which covers are covering invariant. A presheaf of groupoids $\mathcal{M}$ is $n$-algebraic, with $n \geq 3$, if and only if there is a weak equivalence

$$
\pi_{\text {oid }} X_{\bullet} \stackrel{\sim}{\longrightarrow} \mathcal{M} \in P(\mathcal{C}, \mathcal{G r p d})_{L}
$$

where $X_{\bullet} \in \mathcal{C}_{\bullet}$ is a simplicial object such that

(i) $X_{\bullet}$ is a 2-coskeleton.

(ii) The following Kan maps are covers

$$
\begin{gathered}
d_{0}, d_{1}: X_{1} \rightarrow X_{0} \\
X_{2} \rightarrow X_{\bullet}^{\Lambda_{i}^{2}}, i=0,1,2 \\
X_{3} \rightarrow X_{\bullet}^{\Lambda_{i}^{3}}, i=0,1,2,3 .
\end{gathered}
$$

Furthermore, if $\mathcal{M}$ is homotopically discrete and satisfies the above condition then it is in fact 2-algebraic.

As in the case of Artin stacks, an alternate characterization in terms of simplicial sets is: $\mathcal{M}$ is $n$-algebraic if and only if there is $X_{\bullet}$ as above and a weak equivalence

$$
X_{\bullet} \stackrel{\sim}{\longrightarrow} N \mathcal{M} \in P(\mathcal{C}, s \mathcal{S} e t)_{L}
$$

Using the concept of $n$-groupoid, all of the above characterization results can be phrased in a uniform way. Recall that an $n$-groupoid is a simplicial set for which the Kan condition hold in dimensions $\leq n$ and hold with unique lifting for $k>n$. One can define a simplicial object in $\mathcal{C}$ to be an $n$-groupoid if the Kan maps are covers for $k \leq n$ and isomorphisms for $k>n$. Then the various characterizations above say that a stack is $n$-algebraic if it is weakly equivalent in $P(\mathcal{C}, s \mathcal{S} e t)_{L}$ (resp. $\left.P(\mathcal{C}, \mathcal{G r p d})_{L}\right)$ to a simplicial representable object $X_{\bullet}$ (resp. the homotopy colimit of $\left.X_{\bullet}\right)$ which is an $n$-groupoid and a 2-coskeleton. These characterizations indicate that one should call a presheaf of simplicial sets an $n$-algebraic stack if it is weakly equivalent to a simplicial representable $n$-groupoid $X_{\bullet}$.

In section 5 we prove invariance the property of being $n$-algebraic with respect to homotopy orbits under representable group actions.

Theorem 1.8 (Theorem 5.1). Let $G$ be a group object in $\mathcal{C}$ with $G \rightarrow \star$ a cover and let $\mathcal{M} \stackrel{f}{\rightarrow}$ BG be a map. If $\mathcal{N} \rightarrow \mathcal{M}$ denotes the homotopy fiber of $\mathcal{M} \stackrel{f}{\rightarrow} B G$, then $\mathcal{N}$ is $n$-algebraic/ $P$-Artin if and only if $\mathcal{M}$ is.

One of the motivations for this Theorem comes from work in progress with $\mathrm{G}$. Granja on stacks of ruled surfaces. A ruling of a surface $E$ determines a projection $E \rightarrow C$ which is a $\mathbb{P}^{1}$-bundle over a curve $C$. Let $\mathcal{M}$ be the stack classifying families of ruled surfaces corresponding to a fixed curve $C$, Since the ruling is unique, a 
family of such ruled surfaces naturally projects onto an underlying $C$-bundle. This determines a projection map $\mathcal{M} \rightarrow B \operatorname{Aut}(C)$ and the fiber of this map is the stack of $\mathbb{P}^{1}$-bundles over $C$, (which assigns to $X$ the groupoid of $\mathbb{P}^{1}$-bundles over the product $C \times X)$. Using the above Theorem one can show that $\mathcal{M}$ is algebraic since the stack of $\mathbb{P}^{1}$-bundles over $C$ is known to be algebraic (see $[\mathrm{B}]$ ).

1.1. Notations and conventions. By a Grothendieck topology we mean a collection of coverings satisfying certain axioms (see [MM, Definition 2, p.111]), this is sometimes called a basis for a topology. In particular we assume that $\mathcal{C}$ has pullbacks. We write $P(\mathcal{C}, \mathcal{D})$ for the category of presheaves on $\mathcal{C}$ with values in a category $\mathcal{D}$, write $P(\mathcal{C})=P(\mathcal{C}, \mathcal{S e t})$, and $S h(\mathcal{C})$ for sheaves of sets. We will only consider Grothendieck topologies which are subcanonical, that is, where representable functors are sheaves.

If $X$ is an object in $\mathcal{C}$ we use the same letter to denote the corresponding representable sheaf. Given a groupoid object $\left(X_{o}, X_{m}\right)$ we write $d_{0}, d_{1}: X_{m} \rightarrow X_{o}$ for the domain and range maps.

For us a stack is a presheaf of groupoids $F$ on a small site $\mathcal{C}$ which satisfies descent, or "the homotopy sheaf condition". This means that for all covers $\left\{U_{i} \rightarrow\right.$ $X\}$ in $\mathcal{E}$ the canonical map

$$
F(X) \stackrel{\sim}{\longrightarrow} \operatorname{holim}\left(\prod F\left(U_{i}\right) \Rightarrow \prod F\left(U_{i j}\right) \cdots\right)
$$

is an equivalence of groupoids. For a description of the model structure on $\mathrm{Grpd}$ and explicit descriptions of homotopy limits and colimits in Grpd, see [H1].

The category of presheaves of groupoids on a small site $\mathcal{C}$ admits a model category structure denoted $P(\mathcal{C}, \mathcal{G r p d})_{L}$ in which the fibrant objects are the stacks. This model structure is the localization of the projective model structure on the diagram category $P(\mathcal{C}, \mathcal{G r p d})$ with respect to the collection of maps $\left\{U_{\bullet} \rightarrow X\right\}$ where $\left\{U_{i} \rightarrow\right.$ $X\}$ is a cover in $\mathcal{C}$ and $U_{\bullet}$ is the nerve of this cover taken in $P(\mathcal{C}, \mathcal{G r p d})$ via the Yoneda embedding (see [H1]).

We will need the following basic result [H1, Theorem 5.7] characterizing the weak equivalences in $P(\mathcal{C}, \mathcal{G r p d})_{L}$ as the maps $F \stackrel{\phi}{\longrightarrow} G \in P(\mathcal{C}, \mathcal{G} r p d)$ which satisfy the local lifting conditions.

Definition 1.9. A map $F \stackrel{\phi}{\longrightarrow} G \in P(\mathcal{C}, \mathcal{G r p d})$ satisfies the local lifting conditions if:

(1) Given a commutative square



there exists a cover $\left\{U_{i} \rightarrow X\right\}$ and liftings in the diagram as follows




(2) For $A \rightarrow B$, one of the generating cofibrations in Grpd (see [H1, Section 2.1]) $\partial \Delta^{1}=\{\star, \star\} \rightarrow \Delta^{1}$ or $B \mathbb{Z} \rightarrow \star$, given a commutative square,

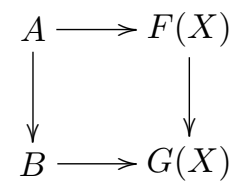

there exists a cover $\left\{U_{i} \rightarrow X\right\}$ and lifts in the diagrams as follows

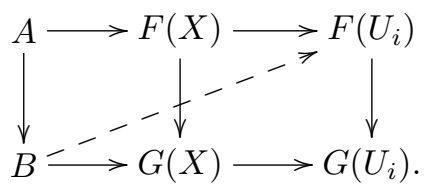

Note that condition (1) means that $F \rightarrow G$ is locally essentially surjective while condition (2) says that $F \rightarrow G$ is locally full and faithful.

The site $\mathcal{A} f f_{\text {flat }}$ consists of affine schemes with covers finite collections of flat morphisms which are jointly surjective. This is sometimes called the fpqc topology. For $(A, \Gamma)$ a Hopf algebroid, $\mathcal{M}_{A, \Gamma}$ denotes a fibrant replacement (or stackification) of the presheaf of groupoids represented by $(\operatorname{Spec} A, \operatorname{Spec} \Gamma)$ in $P\left(\mathcal{A} f f_{\text {flat }}, \mathcal{G r p d}\right)_{L}$.

\section{Properties of Morphisms in $P(\mathcal{C}, \mathcal{G} r p d)_{L}$}

In this section we will define and study properties of morphisms in $P(\mathcal{C}, \mathcal{G r p d})_{L}$. The main Theorem 2.8 describes hypotheses on the property which guarantee that it is a homotopy invariant. We then discuss some immediate consequences of this invariance result.

Definition 2.1. Let $\mathcal{C}$ be a Grothendieck topology and $P$ be a property of morphisms from a sheaf to a representable sheaf.

(1) $P$ is said to be local on the target if $F \stackrel{f}{\longrightarrow} X \in \operatorname{Sh}(\mathcal{C})$ has property $P$ whenever there is a cover $\left\{U_{i} \rightarrow X\right\} \in \mathcal{C}$ such that each $U_{i} \times_{X} F \rightarrow U_{i} \in$ $\mathrm{Sh}(\mathrm{C})$ has property $P$.

(2) $P$ is said to be stable under pullback if given $F \rightarrow X$ with property $P$ and $Y \rightarrow X \in \mathcal{C}$ it follows that $F \times_{X} Y \rightarrow Y$ has property $P$.

We begin by discussing some examples of such properties.

Example 2.2. (i) A map $F \rightarrow X \in \operatorname{Sh}(\mathcal{C})$, with $X \in \mathcal{C}$, is an inclusion if it is a monomorphism of sheaves. The property of being an inclusion is stable under pullback and local on the target.

Proof. The property is clearly stable under pullback. We will show that given $F \rightarrow X$, if $\left\{U_{i} \rightarrow X\right\}$ is a cover and $F_{i}=F \times_{X} U_{i}$ is a subsheaf of $U_{i}$ for each $i$ then $F$ is a subsheaf of $X$. Let $a, b \in F(Z)$ and assume the images of $a, b$ agree in $X(Z)$. Thinking of these as morphisms $a, b: Z \rightarrow F$ we can take pullbacks and obtain maps $a_{i}, b_{i}: U_{i} \times_{X} Z \rightarrow F_{i}$ whose composites with the inclusion $F_{i} \rightarrow U_{i}$ are equal. By hypothesis $F_{i} \rightarrow U_{i}$ is a subsheaf hence 
$a_{i}=b_{i}$. It follows that the images of $a$ and $b$ in each $F\left(U_{i} \times_{X} Z\right)$ agree. As $\left\{U_{i} \times_{X} Z \rightarrow Z\right\}$ is a cover and $F$ is a sheaf, $a$ equals $b$.

(ii) A map of sheaves $f: F \rightarrow X$ is representable if $F$ is isomorphic to a representable functor. This property is always stable under pullback since we are assuming that $\mathcal{C}$ has pullbacks and the Yoneda embedding preserves pullbacks but it is not always local on the target.

Recall [H2, Definition 5.1] that a site $\mathcal{C}$ satisfies descent for morphisms if the presheaf of groupoids $X \mapsto \operatorname{Iso}(\mathcal{C} / X)$ is a stack. If $\mathcal{C}$ satisfies descent for morphisms then representability is a property which is local on the target.

Proof. Suppose we have a cover $\left\{U_{i} \rightarrow X\right\}$ and pullback squares in $\operatorname{Sh}(\mathcal{C})$ :

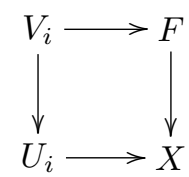

where each $V_{i} \rightarrow U_{i} \in \mathcal{C}$. We have canonical isomorphisms $V_{i} \times_{U_{i}}\left(U_{i} \times_{X} U_{j}\right) \cong$ $V_{j} \times_{U_{j}}\left(U_{i} \times_{X} U_{j}\right)$ over $U_{i} \times_{X} U_{j}$. The universal properties of fiber products guarantee that these isomorphisms satisfy the cocycle condition and so descent for morphisms implies that $F \cong Y$ for some $Y \in \mathcal{C}$.

Note that $\mathcal{A} f f_{\text {flat }}$ - Affine schemes with the flat topology - satisfies descent for morphisms by [SGA, Theorem VIII.2.1].

(iii) A collection $\left\{F_{i} \rightarrow X\right\} \in \mathrm{Sh}(\mathcal{C})$ is a cover if each $F_{i}$ is isomorphic to a representable sheaf $U_{i}$ and the collection of maps $\left\{U_{i} \rightarrow X\right\}$ is a cover in $\mathcal{C}$. Let $\mathcal{C}$ be a site satisfying descent for morphisms. We will say that the topology on $\mathcal{C}$ is local if the property of being a cover is local on the target.

Faithfully flat descent implies that the flat topology on affine schemes $\mathcal{A} f f_{\text {flat }}$ is local and it is easy to see directly that the étale and Zariski topologies on schemes are also local.

(iv) Let $\mathcal{C}=\mathcal{A} f f_{\text {flat }}$. An inclusion $F \rightarrow X$ is an open (closed) subscheme if $F$ is isomorphic to a sheaf represented by an open (closed) subscheme of $X$. These properties are clearly stable under pullback and local on the target.

Note that because an open subscheme of an affine scheme might not be affine, the inclusion of an open subscheme is not necessarily a representable morphism.

(v) Let $\mathcal{C}=\mathcal{A} f f_{\text {flat }}, F \rightarrow X$ is flat if $F$ is represented by a scheme $Y$ and the induced map of schemes $Y \rightarrow X$ is flat. This property is stable under pullback and local on the target.

From now on $P$ will always denote a property of morphisms from sheaves to representable sheaves on $\mathcal{C}$ which is stable under pullback and local on the target.

Definition 2.3. We say $f: \mathcal{M} \rightarrow \mathcal{N} \in P(\mathcal{C}, \mathcal{G r p d})$ is sheaf representable if for all maps $X \rightarrow \mathcal{N}$ with $X \in \mathcal{C}$, the map $X \times{ }_{\mathcal{N}}^{h} \mathcal{M} \rightarrow X$ is weakly equivalent in $P(\mathcal{C}, \mathcal{G r p d})_{L}$ to a map in $\mathrm{Sh}(\mathcal{C})$.

Notice that since sheafification of a presheaf of sets is a weak equivalence in $P(\mathcal{C}, \mathcal{G r p d})_{L}$, sheaf representability is the same as presheaf representability (defined in the obvious way). 
Lemma 2.4. A presheaf of groupoids $F$ is weakly equivalent in $P(\mathcal{C}, \mathcal{G r p d})_{L}$ to a sheaf of sets if and only if the natural maps

$$
F \rightarrow \pi_{0}(F) \rightarrow \operatorname{Sh}\left(\pi_{0}(F)\right)
$$

are weak equivalences.

Proof. The condition is clearly sufficient. Conversely, the local lifting conditions (Definition 1.9) imply that the map $F \rightarrow \operatorname{Sh} F$ is a local weak equivalence for any presheaf of groupoids (see also [H1, Proposition 5.9]).

Thus Sh $F$ is also weakly equivalent to a sheaf of sets. Applying the local lifting conditions to such a zigzag of weak equivalences one easily checks that $\mathrm{Sh} F$ is levelwise homotopically discrete, i.e. $\operatorname{Sh}(F) \rightarrow \pi_{0}(\mathrm{Sh} F)$ is a levelwise weak equivalence.

Using the fact that $\operatorname{Sh}(F)$ is levelwise homotopically discrete, it is easy to check that the map

$$
F \rightarrow \pi_{0}(F)
$$

also satisfies the local lifting conditions.

If $F \stackrel{f}{\longrightarrow} X \in P(\mathcal{C}, \mathcal{G r p d})_{L}$ is a morphism with $X \in \mathcal{C}$ and $F$ is weakly equivalent to a sheaf of sets then the above Lemma provides a functorial factorization of $f$

$$
F \stackrel{\sim}{\longrightarrow} \operatorname{Sh}\left(\pi_{0}(F)\right) \rightarrow X
$$

where the first map is a weak equivalence and the second is a morphism in $\operatorname{Sh}(\mathcal{C})$. It follows that sheaf (or equivalently presheaf) representability of $\mathcal{M} \rightarrow \mathcal{N}$ is equivalent to requiring that $X \times_{\mathcal{N}}^{h} \mathcal{M}$ be weakly equivalent to a presheaf of sets for all maps $X \rightarrow \mathcal{N}$ with $X \in \mathcal{C}$.

If we fix a sheaf representable map $f: \mathcal{M} \rightarrow \mathcal{N}$ then there are factorizations, functorial in maps $X \rightarrow \mathcal{N}$,

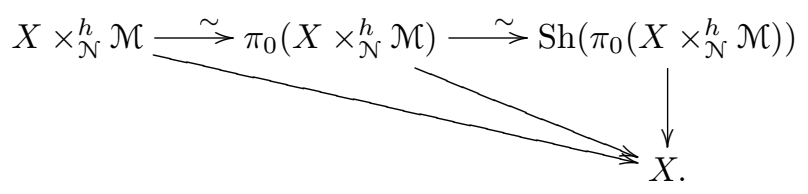

Definition 2.6. Let $P$ be a property. We say that $f: \mathcal{M} \rightarrow \mathcal{N} \in P(\mathcal{C}, \mathcal{G r p d})_{L}$ satisfies property $\mathcal{P}$ if for all maps $X \rightarrow \mathcal{N}$ with $X \in \mathcal{C}$, the map $X \times{ }_{\mathcal{N}}^{h} \mathcal{M} \rightarrow X$ is weakly equivalent to a map in $\mathrm{Sh}(\mathcal{C})$ which satisfies property $P$.

Note that satisfying a property implies sheaf representability and in turn, sheaf representability corresponds to satisfying the empty property of morphisms. Moreover, $\mathcal{M} \rightarrow X$ satisfies property $\mathcal{P}$ iff $\mathcal{M} \rightarrow \pi_{0}(\mathcal{M})$ is a weak equivalence and the induced map of sheaves $\operatorname{Sh}\left(\pi_{0}(M)\right) \rightarrow X$ satisfies property $P$ in the original sense. In particular, for $F \in \mathrm{Sh}(\mathcal{C})$, a map $F \rightarrow X$ satisfies property $P$ in the sense just defined iff it does in the original sense.

Example 2.7. An especially important case is when the property $P$ is having representable domain. Then a morphism $f: \mathcal{M} \rightarrow \mathcal{N}$ satisfying property $\mathcal{P}$ is called a representable morphism.

Notice also that the previous definition makes sense even without the requirement that the property be local on the target and stable under pullback. However when this is not the case then this notion will fail to behave as expected. In particular, being local on the target and stable under pullback guarantees that having property $\mathcal{P}$ is a homotopy invariant in the following sense: 
Theorem 2.8. Let $P$ be a property of morphisms of sheaves with representable target which is stable under pullback and local on the target.

(i) Given a commutative square in $P(\mathcal{C}, \mathcal{G r p d})_{L}$

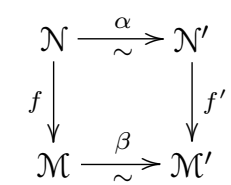

with $\alpha$ and $\beta$ weak equivalences, the morphism $f$ satisfies property $\mathcal{P}$ if and only if $f^{\prime}$ satisfies property $\mathcal{P}$.

(ii) Given a homotopy pullback square in $P(\mathcal{C}, \mathcal{G r p d})_{L}$ with $\beta$ locally essentially surjective



the morphism $f$ satisfies property $\mathcal{P}$ if and only if $f^{\prime}$ satisfies property $\mathcal{P}$.

Proof. We prove (i): it is obvious that $f^{\prime}$ satisfies property $\mathcal{P}$ if and only if $f^{\prime} \circ \alpha=$ $\beta \circ f$ does, so it suffices to see that $f$ satisfies property $\mathcal{P}$ if and only if $\beta \circ f$ does.

Given $X \rightarrow \mathcal{M}, X \in \mathcal{C}$ the map $X \times{ }_{\mathcal{M}}^{h} \mathcal{N} \rightarrow X \times \times_{\mathcal{N}^{\prime}}^{h} \mathcal{N}$ is also a weak equivalence. It follows that if $\beta \circ f$ satisfies property $\mathcal{P}$ then so does $f$.

Now assume $f$ satisfies property $\mathcal{P}$ and $X \rightarrow \mathcal{M}^{\prime}$ is a morphism with $X \in \mathcal{C}$. Using the local lifting conditions one can find a cover $\left\{U_{i} \rightarrow X\right\}$ and for each $i$ a lift making the diagram commute up to homotopy

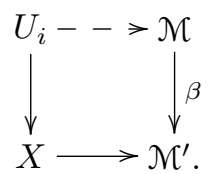

Since $f$ satisfies property $\mathcal{P}$, we see that $U_{i} \times{ }_{\mathcal{M}}^{h} \mathcal{N} \rightarrow U_{i}$ satisfies property $\mathcal{P}$, and since the diagram is homotopy commutative it follows that the pullback of the morphism $X \times{ }_{\mathcal{N}^{\prime}}^{h} \mathcal{N} \rightarrow X$ to $U_{i}$ also satisfies property $\mathcal{P}$.

To finish the proof we must check that, as a property of maps from presheaves of groupoids to representable sheaves, $\mathcal{P}$ is local on the target. For each $i$, we have the following commutative diagram

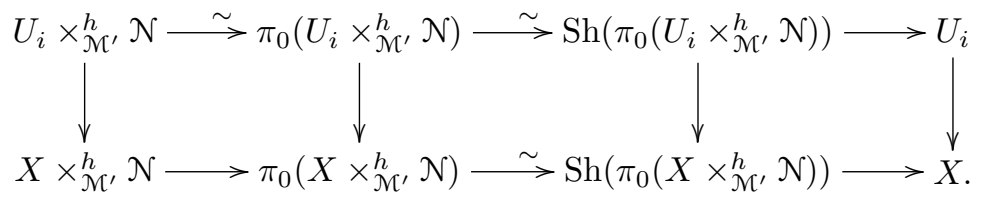

Using our functorial model for the homotopy pullback, the outer rectangle is a pullback square. As sheafification preserves fiber products, the rightmost square must also be a pullback square. As $P$ is local on the target, $\operatorname{Sh}\left(\pi_{0}\left(X \times_{\mathcal{N}^{\prime}}^{h} \mathcal{N}\right)\right) \rightarrow X$ satisfies property $P$ and so it suffices to check that $X \times \times_{\mathcal{M}^{\prime}}^{h} \mathcal{N} \rightarrow \pi_{0}\left(X \times_{\mathcal{M}^{\prime}}^{h} \mathcal{N}\right)$ is a weak equivalence. 
Using the local lifting conditions, it is enough to check that given an automorphism $\alpha \in X \times{ }_{\mathcal{N}^{\prime}}^{h} \mathcal{N}(Z)$, there exists a cover $V_{i} \rightarrow Z$ such that the image of $\alpha$ in each $V_{i}$ is an identity. As $U \rightarrow X$ is locally surjective, $U \times{ }_{\mathcal{N}^{\prime}}^{h} \mathcal{N}^{\prime} \rightarrow X \times_{\mathcal{N}^{\prime}}^{h} \mathcal{N}^{\prime}$ is also locally surjective on automorphisms. Since automorphisms on $U \times_{\mathcal{N}^{\prime}}^{h} \mathcal{N}^{\prime}$ are locally trivial, the same is true on $X \times{ }_{\mathcal{M}^{\prime}}^{h} \mathcal{N}^{\prime}$.

The proof of (ii) is essentially the same as the proof above so we leave it as an exercise for the reader.

Recall that if $\mathcal{C}$ satisfies descent for morphisms then the property of being representable is local on the target (see Example 2.2(ii)).

Corollary 2.9. Let $\mathcal{C}$ be a site satisfying descent for morphisms. Then

(i) The property of being a representable morphism is homotopy invariant in $P(\mathcal{C}, \mathcal{G r p d})_{L}$.

(ii) If in addition the site $\mathcal{C}$ is local (see Example 2.2(iii)) then the property of being a cover is homotopy invariant.

Example 2.10. Let $\mathcal{C}=P\left(\mathcal{A} f f_{\text {flat }}, \mathcal{G r p d}\right)_{L}$. Then being a representable morphism and being a cover are both homotopy invariant notions.

2.1. Generalized algebraic stacks. Deligne and Mumford defined algebraic stacks in $[\mathrm{DM}]$ to study the moduli of curves. Their definition can be naturally abstracted to the following which we recall from [H2].

Definition 2.11. We say that $\mathcal{M} \in P(\mathcal{C}, \mathcal{G} r p d)$ is generalized algebraic if

(i) Its diagonal $\mathcal{M} \rightarrow \mathcal{M} \times \mathcal{M}$ is representable,

(ii) There is a cover $X \rightarrow \mathcal{M}$ with $X \in \mathcal{C}$.

An immediate consequence of Theorem 2.8ii) is:

Lemma 2.12. Let $\mathcal{C}$ be local and satisfy descent for morphisms. If there exists $X \rightarrow \mathcal{M}$ a cover with $X \in \mathcal{C}$, then $\mathcal{M}$ is generalized algebraic.

Remark 2.13. Observe that being generalized algebraic as stated is only a levelwise homotopy invariant. Though $2.11(\mathrm{i})$ is a homotopy invariant of $\mathcal{M}$ if $\mathcal{C}$ satisfies descent for morphisms, 2.11(ii) is only a levelwise homotopy invariant. This subtlety is the source of an inaccuracy in [H2]. The correct statement of the main theorem of [H2] should be that of Theorem 2.15 below (this is actually what is proven in [H2]).

Given a local weak equivalence $\mathcal{M}^{\prime} \rightarrow \mathcal{M}$ the pullback $\mathcal{M}^{\prime} \times{ }_{\mathcal{M}}^{h} X \rightarrow \mathcal{M}^{\prime}$ is a cover in the extended sense but the domain is no longer necessarily a representable. Using the local lifting conditions we can find a cover $\left\{U_{i} \rightarrow X\right\}$ such that each $U_{i}$ factors through $\mathcal{M}^{\prime}$ but this only provides us with a collection of maps

$$
\left\{U_{i} \rightarrow \mathcal{M}^{\prime}\right\}
$$

which is a cover (in the sense that the pullback along representables is a collection of maps weakly equivalent to a cover in $\mathcal{C}$ ), instead of a single map as the definition requires.

Example 2.14. Assume that $\mathcal{C}$ has finite coproducts, and that the collection of maps $\left\{U_{i} \rightarrow \coprod U_{i}\right\}$ is a cover. Let $y$ denote the Yoneda embedding. The natural map

$$
\coprod y\left(U_{i}\right) \rightarrow y\left(\coprod U_{i}\right)
$$


is sheafification and hence is a weak equivalence in $P(\mathcal{C}, \mathcal{G r p d})_{L}$. However, $\amalg y\left(U_{i}\right)$ is not generalized algebraic as there can be no surjective map from a representable $Z$ to it since $\left(\amalg y\left(U_{i}\right)\right)(Z)=\amalg \operatorname{hom}\left(Z, U_{i}\right)$.

Morally speaking, the problem with the definition as stated is that although being a cover is a homotopy invariant being a representable is not. On the subcategory of stacks (fibrant objects in $P(\mathcal{C}, \mathcal{G r p d})_{L}$ ) this problem does not arise since the notions of weak equivalence and levelwise weak equivalence coincide.

The main result of [H2] was a homotopy theoretic characterization of generalized algebraic stacks. Using Theorem 2.8 we can give a very simple proof of this result.

Theorem 2.15. [H2, Theorem 4.3] Let $\mathcal{C}$ be a site which is local and satisfies descent for morphisms. Then $\mathcal{M}$ is a generalized algebraic presheaf of groupoids if and only if there is a weak equivalence

$$
\left(X_{o}, X_{m}\right) \stackrel{\sim}{\longrightarrow} \mathcal{M} \in P(\mathcal{C}, \operatorname{grpd})_{L}
$$

where

- $\left(X_{o}, X_{m}\right)$ is a groupoid object in $\mathrm{C}$,

- the domain map $X_{m} \rightarrow X_{o}$ is a cover.

Proof. Assume $\mathcal{M}$ is generalized algebraic and let $X_{m}$ be the representable weakly equivalent to $X \times{ }_{\mathcal{M}}^{h} X$. Since $X \rightarrow \mathcal{M}$ is a cover it follows from the the local lifting conditions (see Definition 1.9) that the induced map $\left(X, X_{m}\right) \rightarrow \mathcal{M}$ is a weak equivalence.

Conversely, let $\left(X_{o}, X_{m}\right) \rightarrow \mathcal{M}$ be a weak equivalence. One can calculate that the presheaf $\left(X_{o}, X_{m}\right)$ is generalized algebraic and the natural map $X_{o} \rightarrow\left(X_{o}, X_{m}\right)$ a cover. Since $\mathcal{C}$ is local and satisfies descent for morphisms, Corollary 2.9 implies that the properties of being a representable morphism and a cover are homotopy invariant. It follows that the diagonal of $\mathcal{M}$ is also representable and the composite $X_{o} \rightarrow \mathcal{M}$ is a cover.

Example 2.16. The site $\mathcal{C}=\mathcal{A} f f_{\text {flat }}$ is local and satisfies descent for morphisms. It follows that the generalized algebraic presheaves in $P\left(\mathcal{A} f f_{\text {flat }}, G_{\text {rpd }}\right)_{L}$ are the presheaves admitting a weak equivalence from ones that are corepresented by flat Hopf algebroids.

A pair $\left(X_{o}, X_{m}\right)$ as in the statement of the previous Theorem is called a presentation for $\mathcal{M}$. Since $\left(X_{o}, X_{m}\right)$ is cofibrant in $P(\mathcal{C}, \mathcal{G r p d})_{L}$ we can deduce the following Corollary:

Corollary 2.17. Let $\mathrm{C}$ be a site which is local and satisfies descent for morphisms. A presheaf $\mathcal{M}$ is weakly equivalent to a groupoid object $\left(X_{o}, X_{m}\right)$ in $\mathrm{e}$, whose domain map $X_{m} \rightarrow X_{o}$ is a cover, if and only if its fibrant replacement is a generalized algebraic stack.

In particular, this Corollary says that a stack $\mathcal{M}$ is generalized algebraic if and only if it is weakly equivalent to a groupoid object $\left(X_{o}, X_{m}\right)$ in $\mathcal{C}$, whose domain map $X_{m} \rightarrow X_{o}$ is a cover.

When the target is generalized algebraic, there is a simple criterion for checking when a morphism has property $\mathcal{P}$, which follows easily from Theorem 2.8(ii). 
Corollary 2.18. Let $\mathcal{C}$ be a local site satisfying descent for morphisms, $\mathcal{M}$ be generalized algebraic and $X \rightarrow \mathcal{M}$ a cover. A morphism $\mathcal{M}^{\prime} \stackrel{f}{\longrightarrow} \mathcal{M}$ has property $\mathcal{P}$ if and only if the pullback

$$
\mathcal{M}^{\prime} \times_{\mathcal{M}}^{h} X \rightarrow X
$$

is weakly equivalent to a morphism in $\mathcal{C}$ with property $P$.

\section{Algebraic spaces}

Algebraic spaces were introduced by Artin in order to study algebraization problems. We recall the definition.

Definition 3.1. An algebraic space is a sheaf of sets on $\mathcal{A} f f_{\text {ét }}$ which can be written as the coequalizer of two étale maps

$$
R \Rightarrow U
$$

$U, R \in \mathcal{A} f f$, which define a categorical equivalence relation $R \longmapsto U \times U$ (see [Bo]).

The previous definition is the original one given by Artin in [Ar] except that he requires that $R \longmapsto U \times U$ be a closed subscheme, instead of just a monomorphism. Knutson gives a different definition in $[\mathrm{Kn}]$ which is weaker. Although it is phrased slightly differently, it is easy to check that Knutson's definition is equivalent to the above when one replaces $U$ with an arbitrary quasi-separated scheme and require that $R \longmapsto U \times U$ be quasi-compact.

The weakenings of the various definitions are strict. One can see this by considering algebraic spaces which are schemes. Artin's definition guarantees that the scheme is separated and quasi-compact, while the definition above allows for quasi-compact schemes which are not separated and Knutson's definition allows for schemes which are not even quasi-compact.

In terms of the discussion in the previous section an algebraic space can be interpreted in the following way.

Proposition 3.2. Let $\mathcal{C}$ be affine schemes with the étale topology. A sheaf of sets $S$ is an algebraic space if and only if it is a generalized algebraic presheaf of groupoids in the sense of Definition 2.11.

Proof. Suppose $S$ is given by $(U, R)$. Then $(U, R)$ is a groupoid object in $\mathcal{C}$ and, by assumption, the projection maps $R \rightarrow U$ are covers. It follows that $(U, R)$ represents a generalized algebraic presheaf. Since $R$ is an equivalence relation, this presheaf of groupoids is levelwise homotopically discrete and hence weakly equivalent to its $\pi_{0}$ which is in turn equivalent to $S$, (its sheafification).

Let $F$ be a sheaf of sets which is a generalized algebraic presheaf of groupoids. Then there is an affine groupoid scheme $\left(X_{o}, X_{m}\right)$ with $d_{0}, d_{1}: X_{m} \rightarrow X_{o}$ étale covers and a weak equivalence $\left(X_{o}, X_{m}\right) \rightarrow F$. Since $F$ is a sheaf of sets we have that $X_{m} \rightarrow X_{o} \times X_{o}$ is a monomorphism of sheaves in the étale topology and therefore also a categorical monomorphism in affine schemes. Hence $X_{m}$ defines an equivalence relation on $X_{o}$ whose quotient is $F$.

Example 3.3. Let $U=\mathbb{A}_{k}^{1} \backslash\{0\}$ with $k$ a field of characteristic not equal to 2 and consider the $\mathbb{Z} / 2$-action on $U$ given by multiplication by -1 . This gives rise to a groupoid object $(U, \mathbb{Z} / 2 \times U)$ in affine schemes which is homotopically discrete (as there is no isotropy for the action). The corresponding equivalence relation is the inclusion of the diagonal union the anti-diagonal in $U \times U$ and the quotient map is 
the squaring map $\mathbb{A}_{k}^{1} \backslash\{0\} \rightarrow \mathbb{A}_{k}^{1} \backslash\{0\}$. This equivalence relation is closed and the corresponding algebraic space is just the scheme $\mathbb{A}_{k}^{1} \backslash\{0\}$.

Remark 3.4. Recall that a scheme $X$ is separated if the diagonal map $X \rightarrow X \times X$ is a closed embedding. By analogy we say that an algebraic space is separated if the diagonal map is closed (i.e. has the property extending the property of being a closed subscheme). It follows from Corollary 2.18 that an algebraic space is separated iff the map $R \subset U \times U$ is a closed subscheme, and so Artin's definition of algebraic space is just Definition 3.1 plus the condition of being separated.

Example 3.5. Consider the following variation on the above example. $\mathbb{Z} / 2 \times U$ is a coproduct of two copies of $U$ which we label as $U_{0}$ and $U_{1}$. Consider the affine groupoid $\left(U, U_{0} \amalg\left(U_{1} \backslash\{ \pm 1\}\right)\right)$. The equivalence relation this generates is as before with the exception that the points $(1,-1)$ and $(-1,1)$ are missing from $R$. The corresponding algebraic space is again a scheme - a punctured line with a double point - which is not separated.

Example 3.6. Let $k$ be a field of characteristic not equal to 2 and consider the sign action of $\mathbb{Z} / 2$ on $\mathbb{A}_{k}^{1}$. The action groupoid is not homotopy discrete since 0 has $\mathbb{Z} / 2$ isotropy and so does not give rise to an algebraic space.

Instead of the action groupoid we can look at the equivalence relation it determines $R \subset \mathbb{A}_{k}^{1} \times \mathbb{A}_{k}^{1}$ ( $R$ is the union of the diagonal and the anti-diagonal). Since the projection maps $R \rightarrow \mathbb{A}_{k}^{1}$ are not étale this does not give a presentation of an algebraic space. In fact, one can check that the coequalizer of the sheaves $R \rightrightarrows \mathbb{A}_{k}^{1}$ is not an algebraic space.

To obtain an algebraic space we can remove the non-trivial isomorphism of the point 0 in the action groupoids. The corresponding equivalence relation is the map $R^{\prime}=\mathbb{A}_{k}^{1} \amalg \mathbb{A}_{k}^{1} \backslash\{0\} \rightarrow \mathbb{A}_{k}^{1} \times \mathbb{A}_{k}^{1}$ where the first factor is mapped by the diagonal and the second by the anti-diagonal. We will show that this algebraic space is not a scheme.

The map $R^{\prime} \rightarrow \mathbb{A}_{k}^{1} \times \mathbb{A}_{k}^{1}$ factors through $R$ and $R^{\prime} \rightarrow R$ is an epimorphism of schemes, but it is not an epimorphism of sheaves. Since it is an epimorphism of schemes, the coequalizers in schemes of $R \Rightarrow \mathbb{A}_{k}^{1}$ and $R^{\prime} \Rightarrow \mathbb{A}_{k}^{1}$ agree (if they exist). If $k$ is algebraically closed one can check that the coequalizer as a ringed space (and therefore as a scheme) is the squaring map $s: \mathbb{A}_{k}^{1} \rightarrow \mathbb{A}_{k}^{1}$ determined by the map of rings $k[x] \rightarrow k[x]$ sending $x$ to $x^{2}$.

However this is not the coequalizer of $R^{\prime} \Rightarrow \mathbb{A}_{k}^{1}$ in the category of sheaves, since the fiber product of

$$
\mathbb{A}_{k}^{1} \stackrel{s}{\rightarrow} \mathbb{A}_{k}^{1} \stackrel{s}{\leftarrow} \mathbb{A}_{k}^{1}
$$

is not equal to $R^{\prime}$. Therefore the algebraic space determined by $R^{\prime} \rightarrow \mathbb{A}_{k}^{1} \times \mathbb{A}_{k}^{1}$ is not a scheme. Note that this algebraic space is not separated.

3.1. Generalized algebraic spaces. Proposition 3.2 suggests the following generalization of the notion of algebraic space to an arbitrary site.

Definition 3.7. A generalized algebraic space is a generalized algebraic presheaf of groupoids which is a sheaf of sets.

Example 3.8. (1) When $\mathcal{C}=\mathcal{A} f f_{\text {ét }}$ a generalized algebraic space is just an algebraic space as in Definition 3.1.

(2) Artin's definition of algebraic space [Ar] corresponds to a separated algebraic space over $\mathcal{C}=\mathcal{A} f f_{\text {ét }}$ (see Remark 3.4). 
(3) Let $\mathcal{C}$ be the category of schemes with some topology. Recall that a scheme is quasi-separated if the diagonal is quasi-compact. We say that a generalized algebraic space is quasi-separated if its diagonal is quasi-compact in the sense of Definition 2.6. If $\left(X_{o}, X_{m}\right)$ is a presentation for the generalized algebraic space $F$, Corollary 2.18 says that $F$ is quasi-separated if and only if the map $X_{m} \rightarrow X_{o} \times X_{o}$ is quasi-compact. Thus Knutson's definition [Kn] of algebraic space is that of a generalized algebraic space over $\mathcal{C}=\mathcal{S} c h_{e t}$ which is quasi-separated.

(4) Let $\mathcal{C}=\mathcal{A} f f_{\text {flat }}$ denote the site which consists of affine schemes and covers finite collections of jointly surjective flat maps. In this site any cover $\left\{\operatorname{Spec} R_{i} \rightarrow \operatorname{Spec} S\right\}$ can be replaced by a cover consisting of one open $\cup \operatorname{Spec} R_{i} \cong \operatorname{Spec}\left(\prod R_{i}\right) \rightarrow$ Spec $S$. Separated algebraic spaces are those presented by unicursal flat Hopf algebroids [Ra, Appendix A].

Remark 3.9. Schemes are obtained from affine schemes by gluing affine schemes along Zariski opens. Given $X$ and $\left\{U_{i} \rightarrow X\right\}$ a Zariski cover by affine schemes, $X$ is the coequalizer of the discrete groupoid ( $\left.U_{i}, \coprod U_{i j}\right)$. If $X$ is quasi-compact one can assume that the $U_{i}$ form a finite family. Then $\amalg U_{i}$ is again an affine scheme. If $X$ is also separated then the $U_{i j}$ are also affine and hence $X$ is equivalent to a groupoid in affine schemes.

One is led to suspect that schemes are generalized algebraic spaces for the Zariski topology. This is not the case. A Zariski cover $X \rightarrow Y$ is necessarily an isomorphism so a groupoid whose domain map is a Zariski cover is isomorphic to one where all the structure maps are the identity. It follows that the algebraic spaces for affine schemes in the Zariski topology are just the affine schemes.

To obtain the category of schemes as an instance of generalized algebraic spaces over affine schemes one needs a local topology on affine schemes where an epimorphism $\coprod U_{i} \rightarrow X$, with each $U_{i} \rightarrow X$ a Zariski open, is a cover.

For this purpose we define the local Zariski topology $\mathcal{A} f f_{l . Z a r}$ in which a covers are finite collections of jointly surjective maps $\left\{Y_{i} \rightarrow X\right\}$ such that for each $x \in X$ there exists an open affine neighborhood $U_{x}$ such that $Y_{i} \times_{X} U_{x} \cong \coprod_{J} V_{j}$ where each $V_{j}$ is an open affine subset of $U_{x}$ and $J$ is finite.

It is easy to check that this is a local topology which sits between the étale and Zariski topologies and relative to which quasi-compact separated schemes will be algebraic spaces.

We now give conditions on the site $\mathcal{C}$ so that the property of a morphisms of sheaves having domain a generalized algebraic space is local on the target and stable under pullback. This will play a role in the next section when we characterize Artin stacks.

Definition 3.10. We call the site $\mathcal{C}$ stackable if $\mathcal{C}$ is local, satisfies descent for morphisms, and the following condition: Any cover $\left\{U_{i} \rightarrow X\right\}$ has a subcover $\left\{V_{j} \rightarrow X\right\}$ of cardinality $\kappa$ such that

- coproducts of cardinality $\kappa$ exists in $\mathrm{C}$,

- $\coprod_{j} V_{j} \rightarrow X$ is a cover, and

- $\left\{V_{j} \hookrightarrow \coprod_{j} V_{j}\right\}$ is a cover.

The topologies we usually work with on affine schemes, such as the étale or flat (fpqc) topologies are stackable. Here covers are finite collections and finite coproducts exist in $\mathcal{A} f f$. Most topologies on arbitrary schemes are also stackable. 
There one sometimes considers infinite covering families, but infinite coproducts also exist.

Proposition 3.11. Suppose $\mathcal{C}$ is stackable. Let $F \rightarrow X$ be a map of sheaves and $\left\{U_{i} \rightarrow X\right\}$ a cover such that $F \times_{X} U_{i}$ are generalized algebraic spaces, then $F$ is a generalized algebraic space.

Proof. Using the notation of Definition 3.10, let $Y=\amalg_{j} V_{j}$. Then $Y \rightarrow X$ is a cover of $X$ and since $\amalg V_{j}$ is also the coproduct of the sheaves $V_{j}$ we have that $F \times_{X} Y=\coprod_{j} F \times_{X} V_{j}$ is an algebraic space.

Clearly $F \times_{X} Y \rightarrow F$ is a cover in the sense of Definition 2.6. Taking a cover $U \rightarrow F \times{ }_{X} Y$ with $U \in \mathcal{C}$, we have that the composition $U \rightarrow F$ is again a cover and hence $F$ is a generalized algebraic space (see Lemma 2.12).

If $\mathcal{C}$ is stackable, the property of morphisms from sheaves to representables having domain a generalized algebraic space is local on the target. This property is clearly stable under pullback so we can extend it to a homotopy invariant property of morphisms in $P(\mathcal{C}, \mathcal{G r p d})$ (by Theorem 2.8) which is called being representable in algebraic spaces.

Definition 3.12. A map $f: \mathcal{M} \rightarrow \mathcal{N} \in P(\mathcal{C}, \mathcal{G r p d})_{L}$ is representable in algebraic spaces if for all $X \in \mathcal{C}$, the pullback $X \times_{\mathcal{N}}^{h} \mathcal{M}$ is weakly equivalent to a generalized algebraic space.

Since the notion of generalized algebraic space is relative to a site, it will cause no confusion if from now on we abreviate generalized algebraic space by algebraic space.

3.2. Morphisms between algebraic spaces. Certain properties of representable morphisms can be extended to morphisms which are representable in algebraic spaces. As a first step, we define what it means for a morphism from an algebraic space to have property $P$.

Definition 3.13. A property $P$ (always assumed stable under pullback and local on the target) of maps in $\mathrm{C}$ is said to be covering invariant if:

- A map $f: X \rightarrow Y$ has property $P$ whenever there is a cover $\left\{U_{i} \rightarrow X\right\}$ such that the composites $U_{i} \rightarrow Y$ have property $P$.

- If $f: X \rightarrow Y$ has property $P$ and $\left\{U_{i} \rightarrow X\right\}$ is any cover then the composites $U_{i} \rightarrow Y$ have property $P$.

Example 3.14. Suppose $\mathcal{C}=\mathcal{A} f f_{\text {ét }}$. Then the properties of being étale, flat, smooth, finitely presented, finite type are covering invariant.

If $\mathcal{C}=\mathcal{A} f f_{f p q c}$ the property of being flat is a covering invariant.

Definition 3.15. Let $P$ be a property which is covering invariant and $F$ an algebraic space. A map $f: F \rightarrow X$ with $X \in \mathcal{C}$ has property $P$ if for any cover $j: U \rightarrow F$ with $U \in \mathcal{E}$, the composite $f \circ j$ has property $P$.

The above definition is in fact equivalent to one where one only requires the existence of a cover. The proof is a simple exercise.

Lemma 3.16. Let $P$ be a covering invariant property of morphisms in $\mathcal{C}$ and $F$ an algebraic space. A morphism $f: F \rightarrow X$ has property $P$ if and only if there exists a cover $j: U \rightarrow F$ such that $f \circ j$ has property $P$. 
The proof of the following result follows easily from the previous lemma.

Proposition 3.17. Suppose $\mathcal{C}$ is stackable and $P$ is a covering invariant property. Then the property of morphisms in $S h(\mathcal{C})$ with representable target given by

- having domain an algebraic space, and

- having property $P$ in the sense of Definition 3.15

is stable under pullback and local on the target.

It follows that, when $\mathcal{C}$ is stackable and $P$ is a covering invariant of morphisms, then $P$ extends to a homotopy invariant property of morphisms in $P(\mathcal{C}, \mathcal{G r p d})_{L}$ which we call being representable in algebraic spaces with property $\mathcal{P}$.

Remark 3.18. In [Kn] a map $F \rightarrow F^{\prime}$ of algebraic spaces is said to have property $P$ if it lifts to a map with property $P, U \rightarrow U^{\prime}$ between some covers of the respective algebraic spaces (see [Kn, II.2, Definition 2.1]). It's a simple exercise to check that this definition coincides with the definition of property $\mathcal{P}$ when $\mathcal{C}=\mathcal{A} f f_{\text {ét }}$.

\section{Generalized Artin stacks}

In this section we will recall Artin's definition of algebraic stack and present an abstraction of this valid in an arbitrary site. We will then give a homotopy theoretic characterization of the abstracted definition.

Artin defined an algebraic stack [Ar, Definition 5.1] (now called an Artin stack) as a stack over the site $\mathcal{S} c h_{\text {ét }}$ of schemes in the étale topology such that

- the diagonal $\Delta: \mathcal{M} \rightarrow \mathcal{M} \times \mathcal{M}$ is representable in algebraic spaces,

- there is a smooth and surjective map $X \rightarrow \mathcal{M}$ from a scheme $X$.

Artin also imposed some finiteness conditions which come down to working in the subcategory of schemes which are locally of finite type.

Example 4.1. A simple example of such a stack can be obtained from the action of any smooth group scheme $G$ on a scheme $X$. The pair $(X, G \times X)$ is a groupoid object in schemes and so represents a presheaf of groupoids. Let $\mathcal{M}$ denotes the stack associated to this presheaf (i.e. its fibrant replacement in $\left.P\left(\mathcal{S} c h_{\text {ét }}, \mathcal{G} r p d\right)_{L}\right)$. It is easy to compute that $X \times{ }_{\mathcal{M}}^{h} X \simeq G \times X$. Applying Theorem 2.8(ii) we see that the locally essentially surjective morphism $X \rightarrow \mathcal{M}$ is both smooth and representable. We may apply this theorem again to show that the diagonal of $\mathcal{M}$ is representable in $\mathcal{S} c h_{\text {ét }}$.

Replacing $\mathcal{S} c h_{\text {ét }}$ in the above definition with a stackable site $\mathcal{C}$ and smoothness with a covering invariant property $P$ we can abstract the above definition as follows:

Definition 4.2. Let $\mathrm{C}$ be stackable and $P$ be a covering invariant property. We say that $\mathcal{M} \in P(\mathrm{C}, \mathcal{G r p d})$ is $P$-Artin if

- its diagonal is representable in algebraic spaces, and

- there is a map $X \rightarrow \mathcal{M}$ from a representable $X$ which is locally essentially surjective and is representable in algebraic spaces with property $\mathcal{P}$. 
4.1. Characterization of $P$-Artin Stacks. In this subsection we give the characterization of $P$-Artin stacks. We begin with the following Lemma which is an immediate consequence of Theorem 2.8 and Proposition 3.17 (see the proof of Theorem 2.15).

Lemma 4.3. A presheaf $\mathcal{M}$ is $P$-Artin if and only if there is a weak equivalence from a presheaf of groupoids $(X, F) \stackrel{\sim}{\longrightarrow} \mathcal{M}$ with $X$ representable, $F$ weakly equivalent to an algebraic space, and $F \rightarrow X$ having property $P$.

We will need some notation for the statement of our main theorem. Let $\Delta[n] \in$ $s$ Set be the standard $n$-simplex. We write $\Lambda_{i}^{n}$ for the subsimplicial set obtained by removing the $i$-th face from $\Delta[n]$. This can be written as a coequalizer in $s \mathcal{S e t}$ :

$$
\coprod_{0 \leq k<j \leq n, k \neq i \neq j} \Delta[n-2] \Rightarrow \coprod_{0 \leq j \leq n, j \neq i} \Delta[n-1]
$$

Given a simplicial object $X_{\bullet}$ in a category $\mathcal{C}$ with fiber products we will write $X^{\Lambda_{i}^{n}}$ for the equalizer of

$$
\prod_{0 \leq j \leq n, j \neq i} X_{n-1} \Rightarrow \prod_{0 \leq k<j \leq n, k \neq i \neq j} X_{n-2}
$$

dual to (4.4). There are natural maps $X_{n} \rightarrow X^{\Lambda_{i}^{n}}$ given by $\prod_{j \neq i} d_{j}$, which are called Kan maps.

Recall also that given a simplicial set $X, \pi_{o i d} X$ denotes its fundamental groupoid, which is the groupoid with object set $X_{0}$ and morphisms generated by $X_{1}$ subject to the relations

- $s_{0} x=i d_{x}$, for $x \in X_{0}$

- $d_{0} y \circ d_{2} y=d_{1} y$, for $y \in X_{2}$.

The coskeleton, $\operatorname{cosk}_{n}$ is the right adjoint to the forgetful functor $\mathcal{C}_{\bullet}=\mathrm{e}^{\boldsymbol{\Delta}^{o p}} \rightarrow$ $\mathrm{e}^{\boldsymbol{\Delta}_{n}^{o p}}$, where $\boldsymbol{\Delta}_{n}^{o p}$ is the full subcategory of $\boldsymbol{\Delta}$ with objects $\{0, \ldots, n\}$. A simplicial object is called an $n$-coskeleton if it is isomorphic to a diagram in the image of the functor $\operatorname{cosk}_{n}$.

Theorem 4.5. Let $\mathrm{C}$ be a stackable site and $P$ be a covering invariant property. A presheaf of groupoids $\mathcal{M}$ is $P$-Artin if and only if there is a weak equivalence

$$
\pi_{\text {oid }} X_{\bullet} \stackrel{\sim}{\longrightarrow} \mathcal{M}
$$

where $X_{\bullet} \in \mathcal{C}_{\bullet}$ is a simplicial object satisfying

(i) $X_{\bullet}$ is a 2-coskeleton.

(ii) $d_{0}, d_{1}: X_{1} \rightarrow X_{0}$ have property $P$,

(iii) The Kan maps $X_{2} \rightarrow X_{\bullet}^{\Lambda^{2}}$ are covers for $i=0,1,2$,

(iv) The Kan maps $X_{k} \rightarrow X_{\bullet}^{\Lambda_{i}^{k}}$ are isomorphisms for $k>2$ and all $i$.

Proof. Suppose that $\mathcal{M} \in P(\mathcal{C}, \mathcal{G r p d})$ is $P$-Artin and let $X \rightarrow \mathcal{M}$ be a locally essentially surjective map from a representable as in the definition of $P$-Artin. Letting $F=X \times{ }_{\mathcal{M}}^{h} X$ we clearly have that $(X, F)$ defines an object in $P(\mathcal{C}, \mathcal{G r p d})$ which is weakly equivalent to $\mathcal{M}$ in $P(\mathcal{e}, \mathcal{G r p d})$.

Let $Y \rightarrow F$ be a cover. We have two maps $Y \rightarrow X$ given by composition of the cover with the projections $X \times{ }_{\mathcal{M}}^{h} X \rightarrow X$. They are essentially surjective and, by definition of $P$-Artin, have property $P$. Let $X_{0} \rightarrow X$ be a cover such that

$$
X_{0} \rightarrow X \stackrel{\Delta}{\longrightarrow} F
$$


(with $\Delta$ the diagonal) factors as a map $X_{0} \stackrel{\tilde{\Delta}}{\longrightarrow} Y$. Setting $F^{\prime}=X_{0} \times{ }_{\mathcal{M}}^{h} X_{0}$ we again have that $\left(X_{0}, F^{\prime}\right)$ is a presheaf of groupoids coming with a weak equivalence to $(X, F)$. Notice that the natural map $F^{\prime} \rightarrow F$ is a representable morphism and so $X_{1}=F^{\prime} \times{ }_{F} Y$ is representable. Then $X_{1} \rightarrow F^{\prime}$ is a cover, the maps $d_{0}, d_{1}: X_{1} \rightarrow X_{0}$ have property $P$ and there is a common section $s_{0}: X_{0} \rightarrow X_{1}$ which has as factors the diagonal map $X_{0} \rightarrow F^{\prime}$ and $\tilde{\Delta}: X_{0} \rightarrow Y$.

Now let $X_{2}$ and $d_{i}: X_{2} \rightarrow X_{1}$ be defined by requiring that the following be a pullback diagram

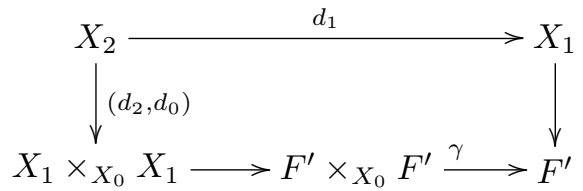

where $\gamma: F^{\prime} \times_{X_{0}} F^{\prime} \rightarrow F^{\prime}$ is the composition map in the groupoid $\left(X_{0}, F^{\prime}\right)$ and $X_{1} \times_{X_{0}} X_{1}$ is the pullback taken along the two projections $X_{1} \rightarrow F^{\prime} \rightarrow X_{0}$. Thus $X_{2}$ consists of lifts to $X_{1}$ of triples formed by a pair of composable arrows in $\left(X_{0}, F^{\prime}\right)$ and its composite.

We can define two degeneracy maps $s_{0}, s_{1}: X_{1} \rightarrow X_{2}$, respectively, as the fiber products of (id, $\left.s_{0} d_{0}\right): X_{1} \rightarrow X_{1} \times_{X_{0}} X_{1}$ (respectively $\left(s_{0} d_{1}\right.$, id)), and the identity map $X_{1} \rightarrow X_{1}$. Clearly the simplicial identities are satisfied in the truncated simplicial object thus defined.

Since $\left(X_{0}, F^{\prime}\right)$ is a groupoid, if in (4.6) we replace the map $\left(d_{2}, d_{0}\right): X_{2} \rightarrow$ $X_{1} \times_{X_{0}} X_{1}$ by either $\left(d_{0}, d_{2}\right)$ or $\left(d_{2}, d_{1}\right)$, the map $d_{1}: X_{2} \rightarrow X_{1}$ by the remaining boundary map, and $\gamma$ by the appropriate operation in the groupoid $\left(X_{0}, F^{\prime}\right)$, then we still obtain a pullback square.

As $X_{1} \rightarrow F^{\prime}$ is a cover, the same is true of the map $X_{2} \rightarrow X_{1} \times_{X_{0}} X_{1}$ in (4.6) which is the Kan map $X_{2} \rightarrow X_{\bullet}^{\Lambda_{1}^{2}}$ in the statement of the Theorem. The argument in the previous paragraph implies that the remaining two Kan maps are also covers.

Finally, we define the simplicial object $X_{\bullet}$ as the 2-coskeleton of the truncated simplicial object defined above (which is possible since $\mathcal{C}$ has pullbacks). Explicitly, $X_{3}$ will consist of lifts to $X_{1}$ of triples of composable maps in the groupoid $\left(X_{0}, F^{\prime}\right)$ together with lifts of all of their composites, and similarly for higher $k$. It is then clear that the Kan maps $X_{k} \rightarrow X_{\bullet}^{\Lambda_{i}^{k}}$ are isomorphisms for $k>2$.

An easy application of the local lifting conditions now shows that the map $\pi_{\text {oid }} X_{\bullet} \rightarrow\left(X_{0}, F^{\prime}\right)$ is a weak equivalence in $P(\mathcal{C}, \mathcal{G r p d})_{L}$, which concludes the proof of necessity.

Now assume that we are given a simplicial object $X_{\bullet}$ satisfying the conditions of the statement. It suffices to show that $\pi_{\text {oid }} X$ • is $P$-Artin, that is, we can take the weak equivalence in the statement of the Theorem to be the identity and $\mathcal{M}=$ $\pi_{\text {oid }} X_{\bullet}$.

Set $F=X_{0} \times{ }_{\mathcal{M}}^{h} X_{0}=\operatorname{mor}(\mathcal{M})$. There is an obvious map $X_{1} \rightarrow F$. As the Kan maps $X_{2} \rightarrow X_{1} \times_{X_{0}} X_{1}$ are covers, they are also epimorphisms of sheaves and so $X_{1} \rightarrow F$ is locally surjective, i.e. $X_{1} \rightarrow \operatorname{sh}(F)$ is an epimorphism of sheaves. By assumption, the two composites $X_{1} \rightarrow F \rightarrow s h(F) \rightarrow X_{0}$ have property $P$ so, by Lemma 4.3 , it remains for us to see that

- $F$ is weakly equivalent to an algebraic space, i.e. $s h(F)$ is an algebraic space, 
- $X_{1} \rightarrow \operatorname{sh}(F)$ is a cover.

By Theorem 2.15 to see that $s h(F)$ is an algebraic space it suffices to check that $X_{1} \times_{s h(F)} X_{1}$ is representable and $X_{1} \times_{s h(F)} X_{1} \rightarrow X_{1}$ is a cover. It then follows that $X_{1} \rightarrow \operatorname{sh}(F)$ is also a cover.

We have the following pullback square

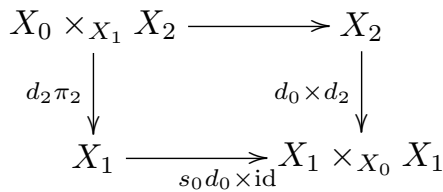

where the top left hand corner is the pullback of the map $d_{0}: X_{2} \rightarrow X_{1}$ and $s_{0}: X_{0} \rightarrow X_{1}$. By assumption, the right hand map (one of the Kan maps) is a cover, so $d_{2} \pi_{2}$ is a cover, and a similar argument shows that $d_{1} \pi_{2}$ is also a cover. The proof will therefore be complete when we prove that

$$
X_{0} \times_{X_{1}} X_{2} \stackrel{\left(d_{1} \times d_{2}\right) \pi_{2}}{\longrightarrow} X_{1} \times_{s h(F)} X_{1}
$$

is an isomorphism, or equivalently, as $X_{1} \times_{s h(F)} X_{1} \cong \operatorname{sh}\left(X_{1} \times_{F} X_{1}\right)$, that

$$
X_{0} \times_{X_{1}} X_{2} \stackrel{\left(d_{1} \times d_{2}\right) \pi_{2}}{\longrightarrow} X_{1} \times_{F} X_{1}
$$

is a local weak equivalence.

Note that $X_{0} \times_{X_{1}} X_{2}$ consists of triangles with degenerate 0 -face. The Kan conditions on $X_{\bullet}$ in dimensions 2 and 3 imply that (4.8) is locally surjective. Concretely, given two 1-simplices in $X_{1}$ with common source which map to the same arrow in $F$, there is locally a 2-simplex in $X_{2}$ with degenerate 0-face having the 1-simplices as its remaining edges.

To see that (4.8) is a monomorphism, notice that two elements in $X_{0} \times_{X_{1}} X_{2}$ with the same image in $X_{1} \times_{F} X_{1}$ are two triangles with the same boundary. Since $X_{\bullet}$ is a 2-coskeleton we can find an element in $X_{3}$ having the given triangles in $X_{2}$ as two of the faces and the two remaining faces degenerate. The assumption that the Kan maps $X_{3} \rightarrow X_{\bullet}^{\Lambda_{i}^{2}}$ are isomorphisms says that the element in $X_{3}$ is determined by any of its horns. Considering the horns obtained by removing the face corresponding to each of the given triangles in $X_{2}$ we see that these must be equal.

In particular, a stack $\mathcal{M}$ is $P$-Artin if and only if it is weakly equivalent to the fundamental groupoid of $X_{\bullet} \in \mathcal{C}_{\bullet}$, satisfying the hypothesis of Theorem 4.5.

Recall that the local model structure $P(\mathcal{C}, s \mathcal{S} e t)_{L}$ can be obtained as a localization of the projective model structure with respect to hypercovers [DHI]. Here weak equivalences can be described in terms of local lifting conditions for presheaves of simplicial sets described in [DI], [DHI]. We will need a special case which applies to locally fibrant simplicial presheaves. A presheaf $F \in P(\mathcal{C}, s \mathcal{S} e t)_{L}$ is locally fibrant if the Kan lifting conditions hold locally, that is, if given a commutative square




there exists a cover $\left\{U_{i} \rightarrow X\right\}$ and lifts in the diagrams as follows

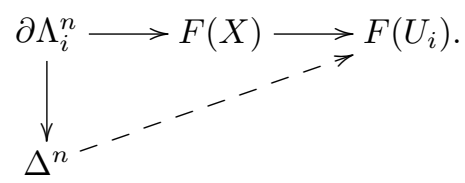

Explicitly we will apply the following:

Proposition 4.9. [DHI, Proposition 3.1] A map $F \rightarrow G$ between locally fibrant simplicial presheaves is a weak equivalence if and only if given a commutative square

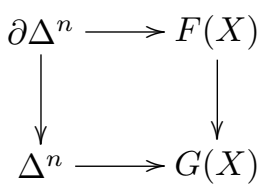

there exists a cover $\left\{U_{i} \rightarrow X\right\}$ and lifts in the diagrams as follows

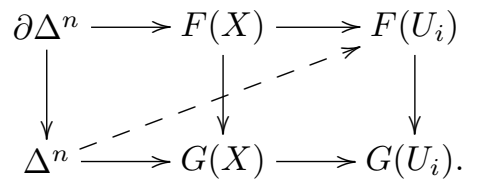

The hypothesis on $X_{\bullet}$ in the statement of Theorem 4.5 guarantees that $X_{\bullet}$ is a locally fibrant presheaf of simplicial sets. The presheaf $N \pi_{\text {oid }} X_{\bullet}$ is levelwise fibrant. One can apply the previous Proposition to see that the map

$$
X_{\bullet} \stackrel{\sim}{\longrightarrow} N \pi_{\text {oid }} X_{\bullet} \in P(\mathcal{C}, s \mathcal{S} e t)_{L}
$$

is a weak equivalence. We obtain as a corollary the following variation on Theorem 4.5 in $P\left(\mathrm{C}, s S_{e t}\right)_{L}$.

Corollary 4.10. A presheaf of groupoids $\mathcal{M}$ is P-Artin if and only if there exists a simplicial representable $X_{\bullet} \in \mathcal{C}_{\bullet}$ satisfying the hypothesis of Theorem 4.5 and a weak equivalence

$$
X_{\bullet} \stackrel{\sim}{\longrightarrow} N \mathcal{M} \in P(\mathcal{C}, s \mathcal{S e t})_{L} .
$$

4.2. Higher analogues of Artin stacks. Let $\mathcal{C}$ be a stackable site (see Definition 3.10). In this section we will define and analyze higher analogues of Artin stacks. For the sake of simplicity we will restrict attention to the case where the property $P$ in Definition 4.2 is simply being a cover, though similar results can be obtained for other covering invariant properties. We necessarily assume throughout this section that to be a cover is a covering invariant property in $\mathrm{C}$.

When $P$ is the property of being a cover we will call a $P$-Artin stack a 2 -algebraic stack. By analogy with the definition of algebraic space we will call a discrete 2algebraic stack a 2-algebraic space. This suggests a hierarchy of definitions which we will now present.

Definition 4.11. Let $\mathcal{M} \in P(\mathcal{C}, \mathcal{G r p d})_{L}$. A map $X \rightarrow \mathcal{M}$ is called a 0 -representable cover if $\mathcal{M} \in \mathcal{C}$ and the map $X \rightarrow \mathcal{M}$ is a cover in $\mathcal{C}$. 
(1) We say that $\mathcal{M}^{\prime} \rightarrow \mathcal{M}$ is an $n$-representable cover, $n>0$, if for all $Y \rightarrow \mathcal{M}$, with $Y \in \mathcal{C}, Y \times_{\mathcal{M}}^{h} \mathcal{M}^{\prime}$ is weakly equivalent to an $(n-1)$-algebraic space and the projection map $Y \times{ }_{\mathcal{M}}^{h} \mathcal{M}^{\prime} \rightarrow Y$ is weakly equivalent to a cover, in the sense of (3) below.

(2) $\mathcal{M}$ is $n$-algebraic, $n \geq 0$, if there exists an $n$-representable cover $X \rightarrow \mathcal{M}$, with $X \in \mathcal{C}$. A sheaf of sets which is $n$-algebraic is called an $n$-algebraic space.

(3) If $F$ is an $n$-algebraic space, $n \geq 0$, a map $F \rightarrow X$, with $X \in \mathcal{C}$, is a cover if for all n-representable covers $Y \rightarrow F$, with $Y \in \mathcal{C}$, the composite $Y \rightarrow F \rightarrow X$ is a cover in $\mathcal{C}$.

Remark 4.12. In the first item of the Definition, requiring that $Y \times_{\mathcal{M}}^{h} \mathcal{M}^{\prime}$ be weakly equivalent to an $(n-1)$-algebraic space is the same as requiring that $Y \times{ }_{\mathcal{M}}^{h}$ $\mathcal{M}^{\prime} \stackrel{\sim}{\sim} \pi_{0}\left(Y \times{ }_{\mathcal{M}}^{h} \mathcal{M}^{\prime}\right)$ be a weak equivalence and $\operatorname{sh}\left(\pi_{0}\left(Y \times{ }_{\mathcal{M}}^{h} \mathcal{M}^{\prime}\right)\right)$ be an $(n-1)$ algebraic space (see Lemma 2.4). It follows that $Y \times{ }_{\mathcal{M}}^{h} \mathcal{M}^{\prime} \rightarrow Y$ is weakly equivalent to a cover if and only if $\operatorname{sh}\left(\pi_{0}\left(Y \times_{\mathcal{M}}^{h} \mathcal{M}^{\prime}\right)\right) \rightarrow Y$ is a cover in the sense of Definition 4.11(3) above.

Since we assume the site $\mathcal{C}$ is local, in Definition 4.11(3) we can replace the condition that the composite be a cover for all covers, with the condition that this happens for some cover.

The initial conditions of the induction imply that 0-algebraic presheaves are true representables. A 1-representable cover is what we have called a representable morphism which is a cover. Hence, 1-algebraic is what we have called generalized algebraic, and a 1-algebraic space is an algebraic space. A 2-representable cover is what we have called a cover represented in algebraic spaces. It follows that a 2-algebraic presheaf is a $P$-Artin presheaf for $P$ the property of being a cover.

The argument used to prove Proposition 3.11 implies that if $\mathcal{C}$ is stackable then the property of maps of sheaves with representable target of having domain an $n$-algebraic space is local on the target (and similarly for the property of being a cover in the sense of Definition $4.11(3))$. The proof goes by induction using the fact that being a cover by an $(n-1)$-algebraic space is local on the target and stable under pullback. The fact that having domain an n-algebraic space is stable under pullback also follows easily by induction on $n$.

We conclude that being an $n$-representable cover is a homotopy invariant property in $P(\mathcal{C}, \mathcal{G r p d})_{L}$. Therefore we have the analogue of Lemma 4.3 and Theorem 2.15 for $n$-algebraic presheaves, as follows:

Lemma 4.13. A presheaf $\mathcal{M}$ is $n$-algebraic if and only if there is a weak equivalence from a presheaf of groupoids $(X, F) \stackrel{\sim}{\longrightarrow} \mathcal{M}$ with $X$ representable, $F$ weakly equivalent to an $(n-1)$-algebraic space, and $F \rightarrow X$ weakly equivalent to a cover.

Recall that, in the above Lemma, $F$ is discrete and so one is in fact requiring that $s h(F)$ be an $(n-1)$-algebraic space, and $s h(F) \rightarrow X$ a cover. The Lemma implies that if $\mathcal{M}$ is a stack, it is $n$-algebraic if and only if it is weakly equivalent to a presheaf of groupoids $(X, F)$ with $X$ representable, $F$ an $(n-1)$-algebraic space, and $F \rightarrow X$ a cover.

We have the following characterization of $n$-algebraic presheaves of groupoids. It implies that if $\mathcal{M}$ is $n$-algebraic with $n>3$, then it is in fact 3-algebraic. 
Theorem 4.14. Let $\mathcal{C}$ be a stackable site and suppose covers are covering invariant in $\mathrm{C}$. A presheaf of groupoids $\mathcal{M}$ is $n$-algebraic, with $n \geq 3$, if and only if there is a weak equivalence

$$
\pi_{\text {oid }} X_{\bullet} \stackrel{\sim}{\longrightarrow} \mathcal{M}
$$

where $X_{\bullet} \in \mathcal{C}_{\bullet}$ is a simplicial object such that

(i) $X_{\bullet}$ is a 2-coskeleton.

(ii) The following Kan maps are covers

$$
\begin{gathered}
d_{0}, d_{1}: X_{1} \rightarrow X_{0}, \\
X_{2} \rightarrow X_{\bullet}^{\Lambda_{i}^{2}}, i=0,1,2 . \\
X_{3} \rightarrow X_{\bullet}^{\Lambda_{i}^{3}}, i=0,1,2,3 .
\end{gathered}
$$

Furthermore, if $\mathcal{M}$ is homotopically discrete and satisfies the above conditions, then it is in fact 2-algebraic.

Remark 4.15. Since $X_{\bullet}$ is a 2-coskeleton, and $s k_{2} \Lambda_{i}^{n} \stackrel{\cong}{\longrightarrow} s k_{2} \Delta^{n}$, for $n>3$, the Kan maps

$$
X_{n} \stackrel{\cong}{\longrightarrow} X_{\bullet}^{\Lambda_{i}^{n}}, n>3
$$

are isomorphisms. It follows that the analogue of hypothesis (iv) of Theorem 4.5 is also satisfied here. One sees that the hypothesis on $X_{\bullet}$ given in the previous Theorem 4.14 and those of Theorem 4.5 fit into a family indexed by an integer $k$ such that the Kan maps are required to be isomorphisms above dimension $k$ and covers up to dimension $k$ (here $k=3$ and in Theorem $4.5 k=2$ ).

Proof. We begin with an $n$-algebraic stack and construct the desired simplicial object. As in the proof of Theorem 4.2 we can construct a truncated simplicial presheaf $\left(F_{2}^{\prime}, Y_{1}, Y_{0}\right)$ whose fundamental groupoid is $\mathcal{M}$ and where $d_{i}: Y_{1} \rightarrow Y_{0}$ are covers. Here $F_{2}^{\prime}$ is defined as the pullback $\left(Y_{1} \times{ }_{Y_{0}} Y_{1}\right) \times{ }_{F^{\prime}} Y_{1}$ where $F^{\prime}=Y_{0} \times{ }_{\mathcal{M}}^{h} Y_{0}$ is $(n-1)$-algebraic and $Y_{1} \rightarrow F^{\prime}$ is an $(n-2)$-representable cover. Now $F_{2}^{\prime}$ is $(n-2)$ algebraic and the covering Kan maps $F_{2}^{\prime} \rightarrow Y_{1} \times_{Y_{0}} Y_{1}$ are $(n-2)$-representable covers.

We can find an $(n-3)$-representable cover $U_{2} \rightarrow F_{2}^{\prime}$, and a cover $U_{1} \rightarrow Y_{1}$ so that the degeneracies $Y_{1} \rightrightarrows F_{2}^{\prime}$ lift to maps $U_{1} \rightarrow U_{2}$. We can then choose a cover $U_{0} \rightarrow Y_{0}$ so that the degeneracy $Y_{0} \rightarrow Y_{1}$ lifts to a map $U_{0} \rightarrow U_{1}$.

Now set $X_{0}=U_{0}, X_{1}=\left(U_{0} \times_{\mathcal{M}}^{h} U_{0}\right) \times_{F^{\prime}} U_{1}$, and $X_{2}=\left(\left(X_{1} \times X_{0} X_{1}\right) \times_{F^{\prime}} X_{1}\right) \times_{F_{2}^{\prime}}$ $U_{2}$. We claim that $X_{2}$ is representable. This is because the map

$$
\left.\left(X_{1} \times_{X_{0}} X_{1}\right) \times_{F^{\prime}} X_{1}\right) \rightarrow F_{2}^{\prime}
$$

is representable, as $F_{2}^{\prime}=\left(Y_{1} \times_{Y_{0}} Y_{1}\right) \times_{F^{\prime}} Y_{1}$ and the map is induced by the maps $X_{0} \rightarrow Y_{0}, X_{1} \rightarrow Y_{1} \in \mathcal{C}$.

One can check that this choice of $X_{i}$ defines a 2-truncated simplicial object in which all the existing Kan maps are covers. Let $X_{\bullet}$ be the 2-coskeleton of this truncated simplicial object. By construction, the fundamental groupoid of $X_{\bullet}$ is weakly equivalent to $\mathcal{M}$. The only thing left for us to check is that the Kan maps out of $X_{3}$ are covers.

Let $F=X_{0} \times{ }_{\mathcal{M}}^{h} X_{0}$ and recall that $X_{1} \rightarrow F$ is a $(n-2)$-representable cover. Let $F_{2}=\left(X_{1} \times_{X_{0}} X_{1}\right) \times_{F} X_{1}$. This consists of the collections of triples of elements of $X_{1}$ which form commutative triangles in the groupoid $\left(X_{0}, F\right)$. Notice that $X_{2} \rightarrow F_{2}$ is an $(n-3)$-representable cover. 
Letting $X_{\bullet}^{\prime}=\operatorname{cosk}_{2}\left(F_{2}, X_{1}, X_{0}\right)$, we have $\left(X_{\bullet}^{\prime}\right)_{3} \cong X_{\bullet}^{\prime \Lambda_{i}^{3}}$. This is because $F_{2}$ consists of lifts to $X_{1}$ of commutative triangles in the groupoid $\left(X_{0}, F\right)$ and to give a collection of such triangles that form the horn of a 3-simplex is to give the 1-skeleton of 3 -simplex ( 6 elements of $X_{1}$ ) such that 3 out of the 4 faces commute in $\left(X_{0}, F\right)$. But this implies that the remaining face commutes also, and so these faces form the boundary of a 3 -simplex in $X_{\bullet}^{\prime}$, which is the same as a 3 -simplex in $X_{\bullet}^{\prime}$, it being a 2-coskeleton.

Since $X_{\bullet}$ is a 2-coskeleton, $X_{3}$ is the pullback of $X_{2}$ with $X_{\bullet}^{\Lambda_{i}^{3}}$ over its boundary $\left(X_{\bullet}\right)^{\partial \Delta^{2}}$. Observe that $F_{2} \hookrightarrow\left(X_{\bullet}\right)^{\partial \Delta^{2}}$ and so $X_{3}$ is also the pullback in the square below



It follows that $X_{3} \rightarrow X_{\bullet}^{\Lambda_{i}^{3}}$ is also an $(n-3)$-representable cover, but since these are actually representables it is just a usual cover in $\mathcal{C}$.

This completes the proof of the first direction.

For the other direction, assume $X_{\bullet}$ is a simplicial object in $\mathcal{C}$ satisfying the hypothesis of the Theorem. It suffices to consider the case when $\mathcal{M}$ is the fundamental groupoid of $X_{\bullet}$ in $P(\mathcal{C}, \mathcal{G r p d})_{L}$. Let $F=X_{0} \times{ }_{\mathcal{M}}^{h} X_{0}$ be the presheaf of morphisms in $\mathcal{M}$. There is an induced map $X_{1} \rightarrow F$ and the composites $X_{1} \rightarrow X_{0}$ are covers, so we need to show that $F$ is weakly equivalent to a 2-algebraic space, that is $\operatorname{sh}(F)$ is a 2-algebraic space and the map $X_{1} \rightarrow \operatorname{sh}(F)$ is a cover. Since $X_{1} \rightarrow \operatorname{sh}(F)$ is an epimorphism, this amounts to showing that $X_{1} \times_{s h(F)} X_{1}$ is a 1-algebraic space (algebraic space) and the projection maps $X_{1} \times_{s h(F)} X_{1} \rightarrow X_{1}$ are covers.

The Kan conditions on $X_{\bullet}$ imply that the first map

$$
X_{0} \times_{X_{1}} X_{2} \rightarrow X_{1} \times_{F} X_{1} \rightarrow X_{1} \times_{s h(F)} X_{1}
$$

sending a 2-simplex with a degenerate $d_{0}$ edge to its two non-degenerate edges is locally surjective. It follows that the composite is an epimorphism of sheaves and we will prove that it is a cover.

Before doing this, observe that the composite of this map with the projection $\pi_{2}$ onto $X_{1}$ fits into a pullback square

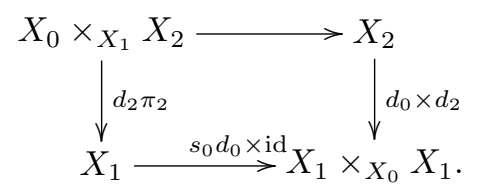

and so $d_{2} \pi_{2}$ is a cover. A similar argument shows that the composite of $\pi_{1}$ with (4.17) is also a cover. It follows that if $X_{1} \times_{s h(F)} X_{1}$ were algebraic then the projections $X_{1} \times{ }_{s h(F)} X_{1} \rightarrow X_{1}$ would be covers, and so we are left to show that (4.17) is indeed a 1-representable cover, i.e. that the fiber product

$$
\left(X_{0} \times_{X_{1}} X_{2}\right) \times_{X_{1} \times{ }_{s h(F)} X_{1}}\left(X_{0} \times_{X_{1}} X_{2}\right)
$$

is representable and the projections onto each of the factors are covers in $\mathcal{C}$. 
Since $X_{1} \times_{s h(F)} X_{1} \hookrightarrow X_{1} \times X_{1}$, the pullback in (4.19) agrees with the pullback taken over $X_{1} \times X_{1}$ and so is representable, and consists of pairs $\left(\sigma_{1}, \sigma_{2}\right)$ of 2 simplices which agree along the boundary and have degenerate 0-edge. We will use this description of (4.19) to build the boundary of a 3-simplex with these faces which will allow us to see that the projections onto the factors are indeed covers.

Taking $s_{1} d_{1} \sigma_{1}$ and $s_{0} s_{0} d_{0} d_{0} \sigma_{1}$ we obtain 2-simplices which, together with $\sigma_{1}$ and $\sigma_{2}$, form the boundary of a 3 -simplex. This construction gives us a pullback square

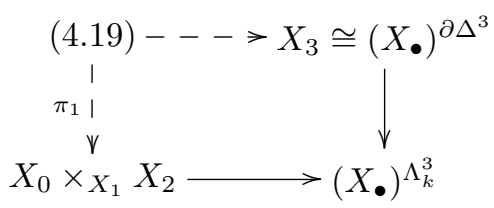

where the bottom horizontal arrow sends $\sigma_{1}$ to the horn formed by $\left(\sigma_{1}, s_{1} d_{1} \sigma_{1}, s_{0} s_{0} d_{0} d_{0} \sigma_{1}\right)$. Since the right hand vertical map is a cover by hypothesis, so is the left vertical map.

This completes the proof of the second direction.

Finally, if $\mathcal{M}$ is locally homotopically discrete, then $\operatorname{sh}(F) \hookrightarrow X_{0} \times X_{0}$ and so $X_{1} \times_{s h(F)} X_{1} \cong X_{1} \times_{X_{0} \times X_{0}} X_{1}$ is representable. We have already shown that the projections $X_{1} \times_{s h(F)} X_{1} \rightarrow X_{1}$ are 1-representable covers, but since the source is representable now it is actually a cover in $\mathcal{C}$. It follows that $\operatorname{sh}(F)$ is an algebraic space and so $\mathcal{M}$ is 2-algebraic.

In particular, a stack $\mathcal{M}$ is $n$-algebraic, $n \geq 3$ if and only if it is weakly equivalent to the fundamental groupoid of $X_{\bullet} \in \mathcal{C}_{\bullet}$, satisfying the hypothesis of Theorem 4.14.

As in Corollary 4.10, the hypothesis on $X_{\bullet}$ together with Proposition 4.9 guarantee that the map $X_{\bullet} \stackrel{\sim}{\longrightarrow} N \pi_{\text {oid }} X_{\bullet} \in P(\mathrm{C}, s \mathcal{S} e t)_{L}$ is a weak equivalence.

Corollary 4.21. A presheaf of groupoids $\mathcal{M}$ is $n$-algebraic, with $n>2$, if and only if there is a weak equivalence

$$
X_{\bullet} \stackrel{\sim}{\longrightarrow} N \mathcal{M} \in P(\mathcal{C}, s \mathcal{S} e t)_{L}
$$

where $X_{\bullet} \in \mathcal{C}_{\bullet}$ is a simplicial object satisfying the hypothesis of Theorem 4.14.

4.3. $\infty$-algebraic stacks. It is natural now to consider the following definition:

Definition 4.22. We say that $X \rightarrow \mathcal{M}, X \in \mathcal{C}$ is an $\infty$-cover if it is locally essentially surjective, and

- setting $\mathcal{M}_{1}=X \times{ }_{\mathcal{M}}^{h} X$, there is a locally essentially surjective map $X_{1} \rightarrow$ $\mathcal{M}_{1}$ with $X_{1} \in \mathcal{C}$ such that the composite $X_{1} \rightarrow X$ is a cover in $\mathcal{C}$.

- Setting $\mathcal{M}_{2}=X_{1} \times{ }_{\mathcal{M}_{1}}^{h} X_{1}$ there is a locally essentially surjective map $X_{2} \rightarrow$ $\mathcal{M}_{2}$ with $X_{2} \in \mathcal{C}$ such that the composite $X_{2} \rightarrow X_{1}$ is a cover in $\mathcal{C}$.

- and so on indefinitely.

We say that $\mathcal{M} \in P(\mathcal{C}, \mathcal{G r p d})_{L}$ is $\infty$-algebraic if it admits an $\infty$-cover.

The argument in the proof of Theorem 4.14 immediately implies the following result.

Corollary 4.23. If $\mathcal{M}$ is $\infty$-algebraic then it is 3 -algebraic. If $\mathcal{M}$ is also homotopically discrete then it is 2-algebraic. 
Example 4.24 (Quasi-compact quasi-separated schemes). Let $\mathcal{C}$ be affine schemes in the local Zariski topology (see Remark 3.9) and let $X$ be a scheme which is quasicompact and quasi-separated. We explained in Remark 3.9 how a quasi-compact separated scheme is an algebraic space in $\mathcal{C}$.

If a scheme is not separated there is no guarantee that intersections of open affines are again affine. However, since $X$ is quasi-separated, intersections of open affines are still quasi-compact, which tells us that $X$ is $\infty$-algebraic in $\mathcal{C}$. By Corollary $4.23, X$ is actually 2 -algebraic. This means that there are local Zariski covers by affine schemes $Y \rightarrow X$ and $Z \rightarrow Y \times_{X} Y$ such that $Z \times_{Y \times{ }_{X} Y} Z$ is affine.

Since every local Zariski cover may be refined by a Zariski cover and $X$ is quasicompact, we may assume that $Y=\amalg U_{i}$ with $\left\{U_{i} \rightarrow X\right\}$ a finite affine cover, and similarly we may assume that $Z=\coprod_{i j k} V_{i j k}$ where $\left\{V_{i j k} \rightarrow U_{i j}\right\}$ are finite affine covers. Then the fact that $Z \times_{Y \times X_{X} Y} Z$ is affine says that the intersections $V_{i j k} \cap V_{i j k^{\prime}}$ are affine for all $i, j, k, k^{\prime}$.

This property of quasi-compact separated schemes is new, to the best of our knowledge.

4.4. $n$-groupoids and stacks in simplicial sets. The characterizations of different sorts of algebraic stacks given above can all be described within a single framework if one considers the concept of an $n$-groupoid.

Definition 4.25. A simplicial set $X_{\bullet}$ is called an $n$-groupoid if

(i) The Kan maps $X_{j} \rightarrow X_{\bullet}^{\Lambda_{i}^{j}}$ are epimorphisms for all $j$ and $i$.

(ii) The Kan maps $X_{m} \stackrel{\cong}{\longrightarrow} X_{\bullet}^{\Lambda_{i}^{m}}$ are isomorphisms for all $m>n$ and all $i$.

Notice that a 1-groupoid is a simplicial set which is the nerve of a groupoid.

Definition 4.26. Let $\mathcal{C}$ be a site. We call a simplicial diagram $X_{\bullet}$ in $\mathcal{C}$ an $n$ groupoid in $\mathcal{C}$, if

(i) The Kan maps $X_{j} \rightarrow X_{\bullet}^{\Lambda_{i}^{j}}$ are covers, for all $j$ and $i$.

(ii) The Kan maps $X_{m} \stackrel{\cong}{\longrightarrow} X_{\bullet}^{\Lambda^{m}}$ are isomorphisms, for all $m>n$ and all $i$.

In light of this, we can reinterpret Theorem 4.2 as saying that the 2-algebraic stacks are those weakly equivalent to 2 -groupoids in $\mathcal{C}$ which are also 2 -coskeletons. Likewise, Theorem 4.14 says that, for $n \geq 3, n$-algebraic stacks are those weakly equivalent to $n$-groupoids in $\mathcal{C}$ which are also 2 -coskeletons. This is in line with what one might expect given the characterization of algebraic (1-algebraic) stacks in terms of groupoids (1-groupoids) in $\mathcal{C}$ (note that groupoids are already 2coskeletons).

If $X_{\bullet}$ is a 2-coskeleton, then being an $n$-groupoid, $n \geq$ is the same as being a 3 -groupoid. This explains why the hierarchy ends at 3 .

If we consider presheaves of simplicial sets, this is no longer the case. These characterizations motivate a definition of an $n$-algebraic m-stack as a presheaf of simplicial sets weakly equivalent to an $n$-groupoid in $\mathcal{C}$ which is also an $m$-coskeleton.

\section{An APPLICATION}

In this section we use Lemmas 4.3 and 4.13 to show that the homotopy orbits of a representable group action on an algebraic stack is again an algebraic stack.

If $G$ is a group object in $\mathcal{C}$ we write $B G$ for a stack weakly equivalent to $(\star, G)$, where $\star \in \mathcal{C}$ denotes the terminal object. 
For the next result we assume as usual that $\mathcal{C}$ is local and satisfies descent for morphisms. If $n>1$, we assume in addition that $\mathcal{C}$ is stackable and that covers and property $P$ are covering invariant.

Theorem 5.1. Let $G$ be a group object in $\mathcal{C}$ with $G \rightarrow \star$ a cover and let $\mathcal{M} \stackrel{f}{\rightarrow}$ BG be a map. If $\mathcal{N} \rightarrow \mathcal{M}$ denotes the homotopy fiber of $\mathcal{M} \stackrel{f}{\rightarrow} B G$, then $\mathcal{N}$ is $n$ algebraic with $n \geq 1$, respectively $P$-Artin, if and only if $\mathcal{M}$ is.

Proof. Given a group $G$ and a fibration of groupoids $H \stackrel{p}{\longrightarrow} G$, there exists a groupoid $F$ and a $G$-action on $F$ so that $p$ admits a functorial weak equivalence from the projection map

$$
G r_{G}(F) \stackrel{p^{\prime}}{\longrightarrow} G
$$

where $G r_{G}(F)$ is the Grothendieck construction for the $G$-action on $F$. Moreover, $p^{\prime}$ is a fibration and so $F$ is both its fiber and homotopy fiber (see [H4, Theorem 2.1] for details).

We may assume $f: \mathcal{M} \rightarrow B G$ is a fibration and $\mathcal{N}$ is its fiber over $\star$ (which is fibrant). Let $\mathcal{M}^{\prime} \rightarrow(\star, G)$ denote the pullback of $f$ along the natural weak equivalence $(\star, G) \rightarrow B G$. Applying the functorial weak equivalence above levelwise, we can find a presheaf of groupoids $F$, an action of the presheaf of groups represented by $G$ on $F$, and a levelwise weak equivalence

$$
G r_{G} F \rightarrow \mathcal{M}^{\prime}
$$

of fibrations over $(\star, G)$. The fiber of $\mathcal{M}^{\prime} \rightarrow(\star, G)$ over $\star$ is also $\mathcal{N}$ and so we have a commutative diagram with weak equivalences as indicated

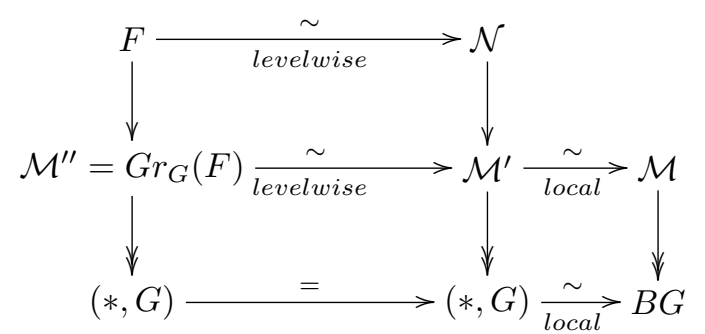

Since $\mathcal{M}^{\prime \prime} \rightarrow \mathcal{M}^{\prime}$ is a levelwise weak equivalence the induced map on fibers $F \rightarrow \mathcal{N}$ is a levelwise weak equivalence also, and so $F$ is also fibrant.

Assuming $\mathcal{N}$ is $n$-algebraic (resp. $P$-Artin) so is $F$, and so there is a representable $X$ and a map $\phi: X \rightarrow F$ which is an $(n-1)$-cover (resp. locally essentially surjective and satisfies property $P$ ). Let $X_{m}=X \times_{F}^{h} X$. Writing $F=\left(F_{o}, F_{m}\right)$, the composite map

$$
X \rightarrow G r_{G} F=\mathcal{M}^{\prime \prime}=\left(F_{o}, F_{m} \times G\right)
$$

is still locally essentially surjective. One can compute that

$$
X \times_{\mathcal{M}^{\prime \prime}}^{h} X \simeq X_{m} \times G
$$

and so $X \times \times_{\mathcal{M}^{\prime \prime}}^{h} X$ is also $(n-1)$-algebraic. The projection map $X \times{ }_{\mathcal{M}^{\prime \prime}}^{h} X \rightarrow X$ factors as the composition $X \times_{\mathcal{M}^{\prime \prime}}^{h} X \rightarrow X \times{ }_{F}^{h} X \rightarrow X$ and hence is an $(n-1)$-cover (resp. locally essentially surjective and satsifies property $P$ ). It follows that $\mathcal{M}^{\prime \prime}$ is $n$-algebraic (resp. $P$-Artin) and hence so is $\mathcal{M}$. 
Conversely, if $\mathcal{M}$ is an $n$-algebraic (resp. $P$-Artin) stack, then there is a representable $X \in \mathcal{C}$ and an $(n-1)$-representable cover (resp. locally essentially surjective map satisfying property $P) X \rightarrow \mathcal{M}$. Since $\star \rightarrow B G$ is representable, the pullback $X \times{ }_{B G}^{h} \star$ is represented by some $E \in \mathcal{C}$. The map $E \rightarrow X$ is a $G$-bundle and is therefore also a cover, since $G \rightarrow \star$ is a cover. It follows that $E \rightarrow \mathcal{M}$ is also a cover (resp. locally essentially surjective and satisfying property $P$ ). Since it factors through $\mathcal{N}$ and $F \stackrel{\sim}{\longrightarrow} \mathcal{N}$ is a levelwise weak equivalence, we may assume that $E$ also factors through $F$.

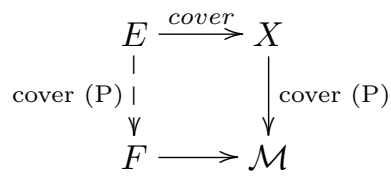

Now one can compute that

$$
\left(E \times_{\mathcal{N}}^{h} E\right) \times G \stackrel{\sim}{\longrightarrow}\left(E \times_{F}^{h} E\right) \times G=E \times_{\mathcal{M}^{\prime \prime}}^{h} E \stackrel{\sim}{\longrightarrow} E \times_{\mathcal{M}}^{h} E
$$

which by hypothesis is $(n-1)$-algebraic and an $(n-1)$-representable cover of $E$ (resp. locally essentially surjective and satisfying property $P$ ). Since the property of being $(n-1)$-representable cover (satisfying property $P$ ) is local on the target, it follows that $E \times_{\mathcal{N}}^{h} E$ is also $(n-1)$-algebraic and is an $(n-1)$-representable cover of $E$ (resp. locally essentially surjective and satisfying property $P$ ).

\section{REFERENCES}

[A] M. Artin, Versal deformations and algebraic stacks, Inventiones Mathematicae 27 (1974), 165-189.

[Ar] M. Artin, Algebraic spaces, James K. Whittemore Lecture in Mathematics given at Yale University, 1969. Yale Mathematical Monographs, 3. Yale University Press, New Haven, Conn.-London, 1971. vii+39 pp.

[B] K. Behrend, PhD Thesis, http://www.math.ubc.ca/ behrend/thesis.html

[Bo] F. Borceux Handbook of Categorical Algebra, University of Cambridge Press, Cambridge UK, 1994.

[DI] D. Dugger and D. Isaksen, Weak equivalences of simplicial presheaves, Homotopy Theory: Relations with algebraic geometry, group cohomology, and algebraic K-theory, 97-113, Contemp. Math. 346, Amer. Math. Soc.

[DHI] D. Dugger, S. Hollander and D. Isaksen, Hypercovers and simplicial presheaves, Math. Proc. Cambridge Philos. Soc. 136 (2004), no. 1, 9-51.

[DM] P. Deligne and D. Mumford, The irreducibility of the space of curves of given genus., Inst. Hautes Études Sci. Publ. Math. 36 (1969), 75-109.

[SGA] A. Grothendieck, Revêtements étales et groupe fondamental, Séminaire de Géométrie Algébrique du Bois Marie 1960-61 (SGA 1), Lecture Notes in Mathematics, Vol. 224, Springer-Verlag, Berlin-New York, 1971.

[H1] S. Hollander, A Homotopy Theory for Stacks., Israel J. Math. 163 (2008), 93-124.

[H2] S. Hollander, Characterizing Algebraic Stacks., Proc. Amer. Math. Soc. 136 (2008), no. 4, $1465-1476$.

[H3] S. Hollander, Geometric criteria for Landweber exactness., Proc. London Math. Soc. 99 (2009) 697-724.

[H4] S. Hollander, Diagrams indexed by Grothendieck constructions., Homology, Homotopy Appl. 10 (2008), no. 3, 193-221.

[Kn] D. Knutson, Algebraic spaces, Lecture Notes in Mathematics, Vol. 203. Springer-Verlag, Berlin-New York, 1971. vi+261 pp.

[LMB] G. Laumon, L. Moret-Bailly, Champs Algébriques, Ergeb. der Math, Vol. 39, Springer Verlag, Berlin, 2000.

[MM] S. MacLane and I. Moerdijk, Sheaves in Geometry and Logic: A First Introduction to Topos Theory, Springer Verlag, Berlin Heidelberg New York, 1992. 
[Ra] D. Ravenel, Complex cobordism and stable homotopy groups of spheres. Pure and Applied Mathematics, 121. Academic Press, Inc., Orlando, FL, 1986. xx+413 pp.

E-mail address: sjh@math.ist.utl.pt+

Center for Mathematical Analysis, Geometry and Dynamical Systems, Instituto Superior Técnico, Tech. Univ. Lisbon, Av. Rovisco Pais, 1049-001 Lisboa, Portugal 